\title{
Relativistic Iron K Emission and absorption in the Seyfert 1.9 galaxy MCG-5-23-16
}

\author{
V. Braito ${ }^{1,2}$, J.N. Reeves ${ }^{1,2,3}$, G.C. Dewangan ${ }^{4}$, I. George ${ }^{1,5}$, R.E. Griffiths ${ }^{4}$, A.

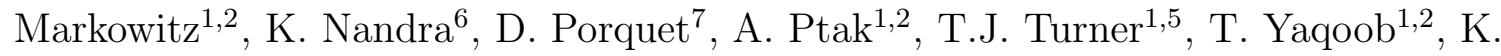 \\ Weaver $^{1}$
}

\begin{abstract}
We present the results of the simultaneous deep XMM-Newton and Chandra observations of the bright Seyfert 1.9 galaxy MCG-5-23-16, which is thought to have one of the best known examples of a relativistically broadened iron $\mathrm{K} \alpha$ line. The time averaged spectral analysis shows that the iron K-shell complex is best modeled with an unresolved narrow emission component (FWHM $<5000$ $\mathrm{km} / \mathrm{s}, \mathrm{EW} \sim 60 \mathrm{eV}$ ) plus a broad component. This latter component has FWHM $\sim 44000 \mathrm{~km} / \mathrm{s}$ and $\mathrm{EW} \sim 50 \mathrm{eV}$. Its profile is well described by an emission line originating from an accretion disk viewed with an inclination angle $\sim 40^{\circ}$ and with the emission arising from within a few tens of gravitational radii of the central black hole. The time-resolved spectral analysis of the $X M M-N e w t o n$ EPIC-pn spectrum shows that both the narrow and broad components of the $\mathrm{Fe} \mathrm{K}$ emission line appear to be constant in time within the errors. We detected a narrow sporadic absorption line at $7.7 \mathrm{keV}$ which appears to be variable on a time-scale of $20 \mathrm{ksec}$. If associated with Fe XXVI Ly $\alpha$ this absorption is indicative of a possibly variable, high ionization, high velocity outflow. The variability of this absorption feature appears to rule out
\end{abstract}

\footnotetext{
${ }^{1}$ Astrophysics Science Division, Code 662, NASA/Goddard Space Flight Center, Greenbelt, MD 20771, USA; vale@milkyway.gsfc.nasa.gov

${ }^{2}$ Department of Physics and Astronomy, Johns Hopkins University, Baltimore, MD 21218.

${ }^{3}$ Astrophysics Group, School of Physical and Geographical Sciences, Keele University, Keele, Staffordshire ST5 5BG

${ }^{4}$ Department of Physics, Carnegie Mellon University, 5000 Forbes Avenue, Pittsburgh, PA 15213

${ }^{5}$ Department of Physics, University of Maryland Baltimore County, 1000 Hilltop Circle, Baltimore, MD 21250, USA

${ }^{6}$ Astrophysics Group, Imperial College London, Blackett Laboratory, Prince Consort Road, London SW7 $2 \mathrm{AW}, \mathrm{UK}$

${ }^{7}$ UMR 7550 du CNRS, Observatoire de Strasbourg, 11 rue de l'Universite, 67000 Strasbourg, France
} 
a local $(z=0)$ origin. The analysis of the XMM-Newton RGS spectrum reveals that the soft X-ray emission of MCG-5-23-16 is likely dominated by several emission lines superimposed on an unabsorbed scattered power-law continuum. The lack of strong Fe L shell emission together with the detection of a strong forbidden line in the O viI triplet is consistent with a scenario where the soft Xray emission lines are produced in a plasma photoionized by the nuclear emission.

Subject headings: galaxies: active - galaxies: individual (MCG-5-23-16) - galaxies: Seyfert - X-rays: galaxies

\section{Introduction}

One of the key issues in high energy research on Active Galactic Nuclei (AGN) is the study of the $6.4 \mathrm{keV}$ iron $\mathrm{K} \alpha$ emission line profile, which can provide fundamental diagnostics of the physical and dynamical conditions of AGN central engines. The fluorescent Fe K $\alpha$ emission line is a prominent and ubiquitous feature in the X-ray spectra of AGN and it is believed to be produced in the innermost regions of the AGN, such as the Broad Line Region (BLR), the circumnuclear obscuring torus and/or the accretion disk. The profile of the line itself provides direct information on the region from which it originates. If the Fe

$\mathrm{K} \alpha$ emission line is produced far from the nucleus, e.g. in the putative torus, its profile is expected to be narrow, while if the line originates in the innermost part of the accretion flow a broad and asymmetric profile is predicted as a result of the special and general relativistic effects such as Doppler shifts, gravitational redshift and light bending (see Fabian et al. 2000; Reynolds \& Nowak 2003 for a review). In the latter case, the shape of the profile itself could be used to derive information on the nature of the black hole and accretion disk system.

The observations with ASCA (Advanced Satellite for Cosmology and Astrophysics) of relativistically broadened iron Fe K $\alpha$ emission lines in AGN (Nandra et al. 1997), and in particular the detection of a broad and skewed profile in the long ASCA observation of MCG-06-30-15 (Tanaka et al. 1995), were considered the first evidence that at least some line emission originates in the inner part of the accretion disk close to the central black hole. However, the scenario emerging from XMM-Newton and Chandra observations of AGN appears to be more complex. Indeed, these observations have shown that only a handful of objects show the presence of the relativistically broadened line, while the narrow Fe emission line at $6.4 \mathrm{keV}$ is an ubiquitous feature in many type I AGN (see Bianchi et al. 2004; Reeves et al. 2004; Yaqoob \& Padmanabhan 2004). Furthermore, the broad component appears to be in general weaker than what was expected given the initial $A S C A$ results and 
in some case it may be absent (i.e. NGC 4151, Schurch et al. 2003). These observations have also shown that the interpretation of the Fe profiles can be strongly dependent on the modeling of the underlying continuum, which can be complicated by the presence of complex absorption (i.e. NGC 3783, Reeves et al. 2004; NGC 3516, Turner et al. 2005) and reflection components (see Reeves et al. 2007 and references therein). Furthermore, red- and blueshifted Fe absorption lines, associated with the presence of infalling or outflowing matter in the proximity of the black-hole, have been detected in the X-ray spectra of QSOs and Seyfert galaxies (see Cappi 2006 and references therein). These absorption and emission features, together with the complexity of modeling the underlying continuum, makes the study of the Fe line profiles more complex and thus feasible only for the brightest objects.

In this framework, MCG-5-23-16 represents one of the best and more robust examples of a relativistically broadened Fe line. MCG-5-23-16 is a nearby $(z=0.008486)$ Seyfert 1.9 galaxy, with a typical 2-10 keV flux of $\sim 8 \times 10^{-11} \mathrm{erg} \mathrm{cm}^{-2} \mathrm{~s}^{-1}$ making it one of the X-ray brightest Seyfert galaxies. Previous X-ray observations showed that the X-ray emission of MCG-5-23-16 resembles the classical spectrum of a Compton thin (i.e. $N_{\mathrm{H}}<10^{24} \mathrm{~cm}^{-2}$ ) Seyfert 2, with a soft excess below $1 \mathrm{keV}$ and a column density $N_{\mathrm{H}} \sim 10^{22} \mathrm{~cm}^{-2}$ (Dewangan et al. 2003; Balestra et al. 2004). Higher energy observations (i.e. above $10 \mathrm{keV}$ ) performed with the Rossi X-Ray Timing Explorer (RXTE; Weaver et al. 1998; Mattson \& Weaver 2004) and BeppoSAX (Perola et al. 2002; Risaliti 2002a) detected a Compton reflection component, that was interpreted as reprocessed emission from the distant molecular torus. A strong broad Fe $\mathrm{K} \alpha$ line was first detected with $A S C A$ (Weaver et al. 1997, 1998) with an equivalent width $\mathrm{EW} \sim 200 \mathrm{eV}$, which could be modeled with a broad relativistic line profile $\left(i \sim 50^{\circ}\right.$, where $i$ is the disk inclination angle) plus a narrow core at $6.4 \mathrm{keV}$ (equivalent width $\mathrm{EW} \sim 60 \mathrm{eV}$ ). The presence of both these components has been subsequently confirmed with Chandra and XMM-Newton observations. In particular, a narrow iron line component at $\sim 6.4 \mathrm{keV}$ has been clearly revealed with the Chandra observation; the intensity of this narrow core (EW $\sim 90 \mathrm{eV}$; Weaver 2001) was found to be in good agreement with the $A S C A$ results. Meanwhile, two short XMM-Newton observations, whose summed exposure time was $\sim 25 \mathrm{ksec}$, confirmed the presence of a underlying broad component with an EW $100 \mathrm{eV}$ (Dewangan et al. 2003; Balestra et al. 2004). However, the relatively short exposure time of the past $X M M-N e w t o n$ observations did not allow the above authors to put strong constraints on the geometry of the emission region. Indeed neither the inner disk radius nor the inclination angle could be accurately derived using only the XMM-Newton data.

In this paper we present an analysis of the iron $\mathrm{K}$ line profile and variability from simultaneous deep XMM-Newton (130 ksec) and Chandra (50 ksec) observations of MCG5-23-16; the analysis and results of the simultaneous deep Suzaku ( 100 ksec) are described 
in Reeves et al. (2007). The XMM-Newton and Chandra observations and data reduction are described in section 2. In section 3 we present the modeling of the time-averaged XMMNewton EPIC and RGS spectra and the results of the spectral fits of the simultaneous Chandra HETG spectra. In section 4 we report the results obtained with time-resolved spectral analysis, aimed to assess the possible variability of the iron $\mathrm{K}$ emission line during the long observation and to investigate the relation (or lack of) between the Fe emission line intensity and the flux of the underlying X-ray continuum. In section 4, we additionally discuss the appearance of a sporadic absorption feature at $7.7 \mathrm{keV}$ (rest-frame) that is indicative of a possibly variable, high velocity $(\mathrm{v} \sim 0.1 c)$ outflow. The results are discussed and summarized in section 5 .

\section{Observations and data reduction}

In December 2005, MCG-5-23-16 was observed simultaneously with many different Xray observatories: Suzaku, XMM-Newton, Chandra and $R X T E$; in Table 1 we report the $\log$ of the different observations. In this paper we concentrate on the XMM-Newton and Chandra observations, while the Suzaku and RXTE observations and results are described in detail by Reeves et al. (2007).

\subsection{XMM-Newton}

MCG-5-23-16 was observed with XMM-Newton on 2005 December 8 for a total exposure time of about $130 \mathrm{ksec}$ (see Table 1). The pn, MOS1 and MOS2 cameras had the medium filter applied; the MOS1 and MOS2 were operating in Small Window mode, while the pn was in Large Window mode. The XMM-Newton data have been processed and cleaned using the Science Analysis Software (SAS ver 6.5) and analyzed using standard software packages (FTOOLS ver. 6.1, XSPEC ver. 11.3). In order to define the threshold to filter for highbackground time intervals, we extracted the 10-12 keV light curves and filtered out the data when the light curve is $2 \sigma$ above its mean. This screening yielded net exposure times (which also includes a dead-time correction) of $96 \mathrm{ksec}, 101 \mathrm{ksec}$ and $103 \mathrm{ksec}$ for the pn, MOS1, and MOS2 respectively. For the scientific analysis we concentrated on the EPIC-pn data, which have the highest signal-to-noise, and we used the MOS1 and MOS2 data to check for consistency. Taking into account the brightness of the source (the $2-10 \mathrm{keV}$ count rates are 7.9 counts $\mathrm{s}^{-1}, 2.7$ counts $\mathrm{s}^{-1}$ and 2.8 counts $\mathrm{s}^{-1}$ for the pn, MOS1, MOS2, respectively), we ran the sas task epatplot to check for possible pile-up and we found that both in the pn and MOS detectors the pile-up fraction is below $1 \%$. However, since we have good photon 
statistics, when analyzing the time averaged pn spectrum, we decided to use only the pattern zero data (which correspond to single events), which are better calibrated, and we considered the pattern 0-4 (single and doubles) when we extract spectra with lower exposure time for the time-variability study.

The EPIC pn source spectrum was extracted using a circular region of $37^{\prime \prime}$ and background data were extracted using two circular regions with an identical radius $\left(37^{\prime \prime}\right)$ centered at $\sim 4^{\prime}$ from the source. EPIC MOS1 and MOS2 data were extracted using a source extraction region of $27^{\prime \prime}$ radius and two background regions with identical size $\left(27^{\prime \prime}\right)$ selected on the nearby CCDs. Response matrices and ancillary response files at the source position have been created using the SAS tasks arfgen and rmfgen. Background subtracted data were then binned to have at least 50 counts in each energy bin.

The Reflection Grating Spectrometer (RGS; den Herder et al. 2001) data have been reduced using the standard SAS task rgsproc and the most recent calibration files; the total exposure times are $\sim 97$ ksec for both RGS1 and RGS2. The RGS1 and RGS2 spectra were binned at the resolution of the instrument $(\Delta \lambda \sim 0.1 \AA)$.

\subsection{Chandra}

Chandra observed MCG-5-23-16 with the ACIS-S with two relatively short exposures for a total of $50 \mathrm{ks}$ (see Table 1). For this study the Chandra observations were made with the High-Energy Transmission Grating (HETG; Markert et al. 1994) in the focal plane of the High Resolution Mirror Assembly. The Chandra HETG consists of two grating assemblies, a High-Energy Grating (HEG) and a Medium-Energy Grating (MEG); the HEG affords the best spectral resolution in the $\sim 6-7 \mathrm{keV}$ Fe-K band currently available $(\sim 39 \mathrm{eV}$, or $1860 \mathrm{~km} \mathrm{~s}^{-1}$ FWHM at $6.4 \mathrm{keV}$ ). The MEG spectral resolution is only half that of the HEG. The HEG also has higher effective area in the Fe K band. The HEG and MEG energy bands are $\sim 0.9-10 \mathrm{keV}$ and $\sim 0.4-8 \mathrm{keV}$ respectively, though the effective area falls off rapidly with energy near both ends of each bandpass.

The Chandra data were reprocessed with CIAO version 3.2.11 and CALDB version 3.0.1. Spectral redistribution matrices ( $\mathrm{rmf}$ files) were made with the CIAO tool mkgrmf for each arm $(-1$ and +1$)$ for the first order data of each of the gratings, HEG and MEG. Telescope effective area files were made with the CIAO script fullgarf which drives the CIAO tool mkgarf. Again, separate files were made for each arm for each grating for the

\footnotetext{
${ }^{1}$ http://cxc.harvard.edu/ciao
} 
first order. The effective areas were corrected for the time-dependent low-energy degradation of the ACIS CCDs using the option available in the mkgarf tool in the stated version of the CIAO and CALDB distribution. Events were extracted from the -1 and +1 arms of the HEG and MEG using strips of width \pm 3.6 arcseconds in the cross-dispersion direction. Lightcurves and spectra were made from these events and the spectral fitting described below was performed on first-order spectra combined from the -1 and +1 orders (using response files combined with appropriate weighting), but keeping the HEG and MEG spectra separate. The background was not subtracted as it is negligible in the energy ranges of interest. Examination of the image of the entire detector and cross-dispersion profiles confirmed that there were no nearby sources contaminating the data.

In the following, unless otherwise stated, fit parameters are quoted in the rest frame of the source and errors are at the $90 \%$ confidence level for one interesting parameter $\left(\Delta \chi^{2}=\right.$ 2.71). Abundances were set to those of Wilms et al. (2000).

\section{Spectral analysis}

\subsection{The $X M M-N e w t o n$ 0.3-10 keV continuum}

To characterize the X-ray continuum of MCG-5-23-16 we first fitted the $2-10 \mathrm{keV}$ pn data with a redshifted power-law model, modified by Galactic $\left(N_{\mathrm{H}}=8 \times 10^{20} \mathrm{~cm}^{-2}\right.$; Dickey \& Lockman 1990) and local absorption. For this initial fit, we ignored the 5.0-7.5 $\mathrm{keV}$ band, where the $\mathrm{Fe} \mathrm{K} \alpha$ emission is expected. This model yielded an acceptable fit $\left(\chi^{2} /\right.$ dof $\left.=1066.3 / 987\right)$ with $\Gamma \sim 1.65$ and $N_{\mathrm{H}} \sim 1.3 \times 10^{22} \mathrm{~cm}^{-2}$. However, the extrapolation of this model to the whole $0.5-10 \mathrm{keV}$ band did not provide a good fit ( $\left.\chi^{2} / \mathrm{dof}=6024.2 / 1794\right)$. Indeed, it left strong residuals in the soft $(\mathrm{E}<1 \mathrm{keV})$ band and, as expected, at the energy of the Fe $\mathrm{K} \alpha$ emission line. Furthermore, it was not clear if the relatively flat photon index was intrinsic or indicative of the presence of emission due to Compton reflection. To illustrate this, in Fig. 1 we show the ratio of the pn data to an absorbed power law model fitted over the $2-10 \mathrm{keV}$ energy band (ignoring the $5.0-7.5 \mathrm{keV}$ band), with the photon index fixed to the best fit value $\Gamma=1.82$, derived with detailed modeling of the XMM-Newton and Suzaku broad band spectra (see Section 3.3).

In order to model the soft X-ray emission, we added to this model a soft power-law component absorbed only by the Galactic column density. The photon index of this soft component was found to be steep, $\Gamma=3.13 \pm 0.10$, and even at the CCD resolution of the pn instrument, the power-law model left line like residuals (see Fig.. 2; black data points). 
In particular an emission line is required by the data $\left(\Delta \chi^{2}=-37\right)$ at $0.92 \pm 0.02 \mathrm{keV}$ with a flux of $1.2 \times 10^{-5}$ photons $\mathrm{cm}^{-2} \mathrm{~s}^{-1}$. This emission line and the steep power-law continuum is confirmed by the MOS1 and MOS2 data (see Fig. 2, red and green data points) and also by the simultaneous Suzaku observation (Reeves et al. 2007). This model gives a $2-10 \mathrm{keV}$ observed flux of $\sim 8.2 \times 10^{-11} \mathrm{erg} \mathrm{cm}^{-2} \mathrm{~s}^{-1}$ and an observed luminosity of $\sim 1.3 \times 10^{43}$ $\operatorname{erg~} \mathrm{s}^{-1}$.

This model is still too simple to describe the overall emission of MCG-5-23-16, because it does not account for the line emission seen in the RGS spectra (see below). However, it demonstrates that scattering of the nuclear power law continuum is a plausible explanation for the soft X-ray continuum spectrum.

\subsection{The RGS spectra: soft X-ray spectrum dominated by emission lines}

In order to investigate if the apparent steep soft X-ray photon-index could be due to the presence of emission lines which are unresolved at the pn CCD resolution, we analyzed the RGS data. Indeed, thanks to the long exposure ( $100 \mathrm{ksec})$, the RGS1 and RGS2 spectra have enough statistics to attempt a spectral analysis (a total of $\sim 17500$ net counts between RGS1 and RGS2). The first inspection of the RGS data reveals the presence of several soft $\mathrm{X}$-ray emission lines as well as the energy cut-off at $\sim 1 \mathrm{keV}$ due to the rest frame absorption. We then rebinned the RGS spectra in constant wavelength bins at the spectral resolution of the instrument $(\Delta \lambda \sim 0.1 \AA)$ and we used the $C$-statistic (Cash 1979) available in XSPEC2 for the spectral fit, because with this choice of binning, we have some bins with less than 20 counts.

We first fitted the RGS spectra with the baseline model obtained with the pn spectrum. This model consists of two components: a primary absorbed power law and a scattered soft power law component absorbed only by Galactic absorption; both photon indices have been fixed to the value found for the primary power law component $(\Gamma=1.82$, see Section 3.3). Overall, this model provides a reasonable description of the RGS continuum, however, linelike residuals are present below $1 \mathrm{keV}$. We then kept this model, hereafter referred as our AGN baseline model and we tested two different additional components for the emission below $1 \mathrm{keV}$, which are: (a) a multi-temperature thermal emission model with variable abundances for different elements (Mewe et al. 1985) or (b) several unresolved emission lines. The first model represents the emission expected from a collisionally ionized plasma; the latter reproduces the emission due to material photoionized by the AGN.

\footnotetext{
${ }^{2}$ http://heasarc.gsfc.nasa.gov/docs/xanadu/xspec/manual/manual.html
} 
When modeling with the multi temperature model, we found that the data could be fitted with two thermal components with $k_{\mathrm{B}} T_{1}=0.44_{-0.14}^{+0.19} \mathrm{keV}$ and $k_{\mathrm{B}} T_{2}=0.15_{-0.06}^{+0.05} \mathrm{keV}$ ( $\Delta C=52$ with respect to the AGN baseline model, for 9 additional parameters). The flux and luminosity of these thermal components are $\mathrm{F}_{(0.5-2) \mathrm{keV}} \sim 9.7 \times 10^{-14} \mathrm{erg} \mathrm{cm}^{-2} \mathrm{~s}^{-1}$ and $\mathrm{L}_{(0.5-2) \mathrm{keV}} \sim 2.2 \times 10^{40} \mathrm{erg} \mathrm{s}^{-1}$, which are consistent with possible X-ray emission from the galaxy. The data below $1 \mathrm{keV}$ still required a contribution from the scattered power law component of the AGN baseline model, which has $\mathrm{F}_{(0.5-2) \mathrm{keV}} \sim 2.1 \times 10^{-13} \mathrm{erg} \mathrm{cm}^{-2} \mathrm{~s}^{-1}$ Allowing the photon index of this soft power law to vary, we found that it was no longer unusually steep $(\Gamma \sim 2.1)$. Although not well constrained, the abundances for $\mathrm{N}, \mathrm{O}$ and Ne required with this model are found to be low, $Z \sim 0.2 Z_{\odot}\left(Z_{N} \sim 0.2 Z_{\odot}, Z_{O} \sim 0.2 Z_{\odot}\right.$, $\left.Z_{\mathrm{Ne}} \sim 0.4 Z_{\odot}\right)$. In particular iron is found to be underabundant, with only an upper limit of $0.2 Z_{\odot}$. This is due to the lack of Fe L shell emission lines and is at odds with the flux measured for Fe K $\alpha$ emission line (see Section 3.3 and Table 3). Furthermore this value is also in contrast with the Fe abundance measured with the neutral iron edge from the reflection component in the simultaneous Suzaku data $\left(Z_{F e}=0.4 \pm 0.1 Z_{\odot}\right.$ at the $90 \%$ confidence level; Reeves et al. 2007).

We then fitted the RGS spectra by adding to the AGN baseline continuum model several unresolved emission lines, fixing both the soft and the hard power-law photon indices to the value found for the AGN primary power-law component $(\Gamma=1.82$, see Section 3.3). To account for the excess of counts below $1 \mathrm{keV}$, five lines are required $(\Delta C=70)$; their fluxes are listed in Table 2, together with their EWs which range from $\sim 10 \mathrm{eV}(\mathrm{N}$ VII $)$ to $\sim 46 \mathrm{eV}$ (O viI). The most likely identifications are $2 \rightarrow 1$ emission lines from $\mathrm{H}$ and He-like $\mathrm{O}, \mathrm{Ne}$ and $\mathrm{N}$ (see Fig. 3).

We tentatively allowed the lines' width to vary and found the O VII He $\alpha$, the O vII radiative recombination continuum (RRC), and the Ne Ix He $\alpha$ lines to be marginally resolved, while the $\mathrm{N}$ VII Ly $\alpha$ and the $\mathrm{O}$ VIII Ly $\alpha$ lines are unresolved. Although a quantitative measure is beyond the statistics of the present data, from the width of the O VII RRC feature (E> $0.739 \mathrm{keV}$ ) we derived an upper limit of $k_{\mathrm{B}} T<24 \mathrm{eV}$ on the recombining electron temperature. It is worth noting that this low value indicates that the soft $\mathrm{X}$-ray emission originates in a photoionized rather than in a collisionally ionized plasma (Liedahl \& Paerels 1996; Liedahl 1999). Taking into account the brightness of the O VIII and the N vII Ly $\alpha$ line, this photoionized material should also produce RRC features from these ions. Neither the O VIII $(\mathrm{E}>0.871 \mathrm{keV})$ or the N VII $(\mathrm{E}>0.667 \mathrm{keV})$ RRC are clearly detected in the RGS spectra. However, the upper limit on the fluxes of both these features are fairly high $\left(5.9 \times 10^{-6} \mathrm{ph}\right.$ $\mathrm{cm}^{-2} \mathrm{~s}^{-1}$ and $3.8 \times 10^{-6} \mathrm{ph} \mathrm{cm}^{-2} \mathrm{~s}^{-1}$ for O VIII and $\mathrm{N}$ VII RRCs respectively). In particular at the energy of the $\mathrm{O}$ VIII RRC feature the continuum of MCG-5-23-16 shows a steep rise due to the emergence of the absorbed power-law component, which also complicates a correct 
deblending of the Ne Ix He $\alpha$ triplet (see below).

The O vir He $\alpha$ and the Ne Ix He $\alpha$ lines are both triplets, which with the present statistics, can not be resolved into their forbidden and resonance components. However it is worth noting that for both the O VII He $\alpha$ and the Ne Ix He $\alpha$ lines the energy centroids are close to the energy of the rest-frame forbidden line (see Table 2). This suggests a major contribution from forbidden lines in each of the triplets with respect to the resonance lines.

In the case of $\mathrm{NeIx} \mathrm{He} \alpha$, its line energy is close to the low energy photoelectric cutoff present in the MCG-5-23-16 spectrum due to the local absorption $\left(N_{\mathrm{H}} \sim 10^{22} \mathrm{~cm}^{-2}\right)$. Furthermore the energy is also close to the O VIII RRC $(>0.871 \mathrm{keV})$ and to the Fe XVIII- XIX 3d-2p blend (0.853-0.926 keV). We then included in the model three more Gaussian lines to account for the O VIII RRC feature and for the decomposition of the Ne IX He $\alpha$ triplet 3 . The fit did not statistically improve, and we cannot derive any quantitative information on the ratio between the intensity of the forbidden and resonance line. In the case of the O VII He $\alpha$ we measured a width of $\sigma=4.6_{-3.1}^{+8.9} \mathrm{eV}$, which is probably due to the presence of the intercombination and resonance component. In order to confirm that the detected emission is dominated by the forbidden line, we then added two lines and kept the line energies frozen for the forbidden $(0.561 \mathrm{keV})$, the intercombination $(0.569 \mathrm{keV})$ and the resonance $(0.574 \mathrm{keV})$ lines. The measured ratio between the flux of the forbidden and resonance lines (for the latter we use the $90 \%$ upper limit) is $\gtrsim 1.6$; which again is evidence of a strong contribution from a photoionized plasma (Porquet \& Dubau 2000). Furthermore with this model we found that the width of the forbidden line is now unresolved $(\sigma<4.7 \mathrm{eV})$. Finally an inspection of the values of the centroid energies of the detected lines (see Table 2 ) shows that there are no strong shifts between the theoretical and observed values. The measured shifts of these lines give a value of $\lesssim 1 \mathrm{eV}$ (which for example for the O viII Ly $\alpha$ corresponds to a velocity $\left.\lesssim 500 \mathrm{~km} \mathrm{~s}^{-1}\right)$.

In order to test whether these lines can be explained with emission from optically thin gas photoionized by the AGN, we replaced the unresolved emission lines with a grid of photoionized emission models generated by XSTAR (Bautista \& Kallman 2001), which assumes a $\Gamma \sim 2$ illuminating continuum and a turbulence velocity of $\sigma_{\mathrm{v}}=100 \mathrm{~km} \mathrm{~s}^{-1}$. We found that the RGS data are well explained with this model with an ionization parameter $\log \xi=1.29_{-0.13}^{+0.17}$. We then let the photon index of the scattered power-law vary and we found that, although it is not well constrained, the value is now similar $\left(\Gamma=1.97_{-0.53}^{+0.18}\right)$ to the primary AGN power law emission, in agreement with the scattering hypothesis. Though

\footnotetext{
${ }^{3}$ The energy of the forbidden $(0.905 \mathrm{keV})$, the intercombination $(0.915 \mathrm{keV})$ and the resonance $(0.922$ $\mathrm{keV}$ ) components were fixed.
} 
even with this model the abundances cannot be constrained, iron $\left(Z_{\mathrm{Fe}}=1.1_{-0.8}^{+1.2} Z_{\odot}\right)$ is not underabundant with respect to the value obtained with the detailed fitting of the $\mathrm{Fe} \mathrm{K} \alpha$ line and the Compton reflection hump detected with the Suzaku observation (Reeves et al. 2007).

As a final test, we applied this best fit model to the pn, MOS1 and MOS2 data. We kept the abundances fixed to the values measured with the RGS spectra and we let only the normalization and the photon index vary. This model is now a good description of the soft spectrum and no strong residuals are present. Finally with this model we found that the scattered component has a $\Gamma=2.3 \pm 0.3$ and $\mathrm{F}_{(0.5-2) \mathrm{keV}} \sim 4 \times 10^{-13} \mathrm{erg} \mathrm{cm}^{-2} \mathrm{~s}^{-1}$. This corresponds to $0.5 \%$ of the un-absorbed flux of the primary AGN component and the emission due to the photoionized gas $\left(\mathrm{L}_{(0.5-2) \mathrm{keV}} \sim 10^{40} \mathrm{erg} \mathrm{s}^{-1}\right)$ is $0.1 \%$ of the AGN emission.

To conclude, although from a statistical point of view either the multi-temperature thermal emission model or the photoionized plasma model (XSTAR) give similar results, different diagnostics suggest that, as already seen in other Seyfert2 galaxies (Bianchi et al. 2006; Guainazzi \& Bianchi 2007; Iwasawa et al. 2003) the soft X-ray emission is likely due to photoionized plasma which could be associated with the Narrow Line Region. The properties of the soft X-ray emission of MCG-5-23-16 that favor this interpretation are: the lack of a strong Fe-L shell emission (Kallman et al. 1996), the detection of a narrow RRC feature from $\mathrm{O}$ VII and the stronger contribution from the forbidden line in the O VII He $\alpha$ triplet.

In order to assess the extent of the soft flux, we used the Chandra observations and created an image combining the $0.3-1.0 \mathrm{keV}$ photons from the zero-order of both observations. We created a point-source function (PSF) using the MARX Chandra simulator. We then fit a model to the Chandra image consisting of a constant component, to account for the background, and two Gaussian components with the centroid positions tied together, to account for both nuclear and extranuclear emission. The model was convolved with the PSF and then compared to the data using the Cash statistic. Initially the image was fit with $\sigma_{x}=\sigma_{y}$, i.e., circular Gaussian models. This gave $\sigma=0.39_{-0.15}^{\prime \prime 0.23}$ for the nuclear component and $\sigma=1.57_{-0.31}^{\prime+0.78}$ for the extranuclear component. The count rates for the two components

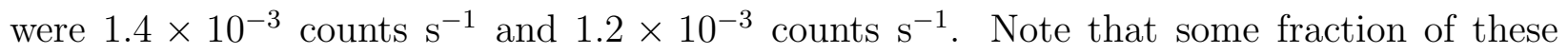
extents is likely due to residual error in the aspect solution, which would effectively increase the PSF. Unfortunately there are no other on-axis point sources bright enough in the field to check this. Allowing the extra-nuclear component to be elliptical did not improve the

\footnotetext{
${ }^{4}$ http://space.mit.edu/CXC/MARX/
} 
fit significantly, however it reduced $C$ by 7.2 for 2 additional parameters (the additional $\sigma$ parameter and the rotation angle), which is significant at the $2 \sigma$ level. It is likely, however, that this asymmetry may be due to aspect errors. As a consistency check we fitted the zero-order image from the 2000 Chandra observation with the same model. In this case allowing for ellipticity in either component did not improve the fit significantly. In this fit the best-fitting parameters were $\sigma=0.56_{-0.12}^{\prime \prime+0.09}$ for the nuclear component and $\sigma=2.2^{\prime \prime+0.5}$ for the extra-nuclear component, which is consistent within the errors. This is indicative that around half of the soft X-ray emission is due to the central point like source and half to an extended component. Assuming the current cosmology $\left(\mathrm{H}_{0}=71 \mathrm{~km} \mathrm{~s}^{-1} \mathrm{Mpc}^{-1}, \Omega_{\Lambda}=0.73\right.$, and $\left.\Omega_{m}=0.27\right)$ the soft X-ray emission of this latter component originates within $\sim 0.7 \mathrm{kpc}$; this value is in agreement with a possible association with the NLR; indeed this extension is comparable to the extension of the [O III] $\lambda 5007$ derived with HST data (Ferruit et al. 2000).

\subsection{The iron $\mathrm{K}$ band}

We then considered the hard X-ray emission of MCG-5-23-16, using the dual power law continuum as described above and only the Gaussian emission line at $\sim 0.9 \mathrm{keV}$. In Fig. 4 we show the residuals left by the absorbed power law model (with $\Gamma=1.65$ ) at the energy of Fe $\mathrm{K}$ band. These residuals clearly reveal the presence of a strong narrow core at the expected energy of the Fe $\mathrm{K} \alpha(6.4 \mathrm{keV})$ and broad wings, which extend from $\sim 5.7 \mathrm{keV}$ to $\sim 7 \mathrm{keV}$. The pn data also show a narrow emission line at $\sim 7 \mathrm{keV}$, due to $\mathrm{Fe} \mathrm{K} \beta$ and a drop at $7.1 \mathrm{keV}$ probably due to a reflection edge. The presence of this latter component was already suggested with the previous short XMM-Newton observations (Dewangan et al. 2003 ), however the short exposure of these observations together with the lack of any simultaneous observation above $10 \mathrm{keV}$ did not allow the authors to put strong constraints on this feature. We adopted the best-fit model obtained by Reeves et al. (2007) from the simultaneous Suzaku observation for the underlying continuum in order to derive the Fe $\mathrm{K} \alpha$ emission line properties. Indeed Suzaku's broad band energy range (0.4-100 keV) allowed these authors to measure the amount of Compton reflection and thus better constrain the continuum in the $2-10 \mathrm{keV}$ energy band. This model is composed of: a primary absorbed power-law component with an exponential cut-off at high energies $(>200 \mathrm{keV})$ and a component due to reflection from neutral material (the PEXRAV model in XSPEC, Magdziarz \& Zdziarski 1995). The parameters of this reflection component are: the reflection fraction, which is defined by the subtending solid angle of the reflector $R=\Omega / 2 \pi=1.1$; an inclination angle $i=45^{\circ}$ and abundance $Z=0.4 Z_{\odot}$. When fitting this model to the pn data we kept the values of $Z, \mathrm{R}$ and the cut-off energy fixed, since they cannot be determined using the lower 
energy band pass of XMM-Newton. After including the reflection component, the residuals no longer show the deep edge at $7.1 \mathrm{keV}$, which is well modeled with the reflection component (see Fig. 5; upper panel). With this model we found that the primary power-law component has a photon index $\Gamma=1.82 \pm 0.01$, absorbed by a neutral column density of $N_{\mathrm{H}}=1.49 \pm 0.01 \times 10^{22} \mathrm{~cm}^{-2}$.

To model the Fe line we first added narrow Gaussian lines at the energies of $\mathrm{Fe} \mathrm{K} \alpha$ and $\mathrm{Fe}$ $\mathrm{K} \beta$. For this latter line we kept the energy fixed at $7.06 \mathrm{keV}$ and tied its flux to be $12 \%$ of the Fe K $\alpha$ flux. This model clearly leaves an excess of counts at the energy of the 6.4 keV Fe K $\alpha$ line (see Fig. 5; middle panel), which can be accounted for by including a broad Gaussian line or a relativistic disk-line component (see section 3.3.2).

\subsubsection{Chandra Observation of the Narrow Core.}

In order to measure the parameters (i.e. strength and profile) of the broad component we first derived the width and flux of the narrow core using the simultaneous Chandra observations. We combined the Chandra \pm 1 MEG and HEG 1st order spectra of both observations. The combined spectra were rebinned at the maximum spectral resolution of the instruments $(\Delta \lambda=0.012 \AA$ and $0.023 \AA$ for HEG and MEG respectively) and the spectral fit was minimized with the $C$-statistic (Cash 1979).

We then adopted the Suzaku best fit model for the underlying 2-8 keV continuum. Thanks to the high resolution of the MEG and HEG instruments, the Chandra HETG residuals clearly reveal the presence of a narrow core at $6.4 \mathrm{keV}$ (see Fig. 6), which is best modeled with a Gaussian line at $6.40_{-0.01}^{+0.02} \mathrm{keV}$ and $\mathrm{EW} \sim 80 \mathrm{eV}(\Delta C=43)$. With this model we measured a width of $\sigma=32_{-16}^{+19} \mathrm{eV}$, which corresponds to a velocity width of $\sigma_{\mathrm{v}} \sim 1400 \mathrm{~km}$ $\mathrm{s}^{-1}$ and is thus indicative of a possible origin from the molecular torus.

Taking into account that the measured width of the Fe K $\alpha$ narrow core could be due to the presence of the broad component, we added a second Gaussian line. Although the fit did not statistically improve, we found an $\mathrm{EW}$ of $\sim 60 \mathrm{eV}$ (flux $\sim 5.6 \times 10^{-5}$ photons $\mathrm{cm}^{-2} \mathrm{~s}^{-1}$; $\sigma \sim 0.4 \mathrm{keV}$ ) and $\sim 70 \mathrm{eV}$ (flux $\sim 5.8 \times 10^{-5}$ photons $\mathrm{cm}^{-2} \mathrm{~s}^{-1}$ ) for the broad and narrow components respectively, which are in good agreement with the values measured with the XMM-Newton (see Table 3) and Suzaku spectra (Reeves et al. 2007). With this model the narrow core is no longer resolved and we can place only an upper limit on the width of $\sigma<43 \mathrm{eV}$, which corresponds to a FWHM $<5000 \mathrm{~km} \mathrm{~s}^{-1}$ (at the $90 \%$ confidence level) in good agreement with the upper limit measured with a previous Chandra observation of MCG-5-23-16 (Balestra et al. 2004). 


\subsubsection{The broad Fe line}

We then adopted the Chandra upper limit on the width of the narrow core for the $X M M-N e w t o n$ fits. The broad component was first modeled adding a second Gaussian line. We also added a Compton shoulder at $6.3 \mathrm{keV}$, with its normalization set to $20 \%$ of the Fe K $\alpha$ flux (Matt 2002); the fit improved with a $\Delta \chi^{2}=44$ for 3 additional parameters $\left(\chi^{2}=1932\right.$ for 1785 dof $)$. The broad Gaussian component $\left(\mathrm{E}=6.22_{-0.16}^{+0.11} \mathrm{keV}\right)$ has an EW of $66_{-18}^{+19} \mathrm{eV}$ and a width of $\sigma=0.42_{-0.10}^{+0.14} \mathrm{keV}$; which corresponds to a velocity of $\sigma_{v} \sim 20000$ $\mathrm{km} \mathrm{s}^{-1}\left(\mathrm{FWHM} \sim 40000 \mathrm{~km} \mathrm{~s}^{-1}\right)$.

We then tested a relativistic diskline model (DISKLINE in XSPEC; Fabian et al. 1989); this code models a line profile from an accretion disk around a Schwarzschild black hole. The main parameters of this model are the inner and outer radii of the emitting region on the disk, and its inclination. The disk radial emissivity is assumed to be a power law, in the form of $r^{-q}$. For the fit we fixed the outer radius to be $400 \mathrm{R}_{\mathrm{g}}$ (with $R_{\mathrm{g}}=G M / c^{2}$ ) and the emissivity $q=3$. Finally we assumed the line to be from neutral Fe K $\alpha$. From a statistical point of view this fit gives a similar result to the model with a broad Gaussian line ( $\chi^{2}=1928$ for 1785 dof), however the high velocity inferred from the width of the Gaussian line is indicative that the line must be produced close to the central black hole; i.e. within $100 \mathrm{R}_{\mathrm{g}}$ and thus inside the Broad Line Region. With this model we found that the inner radius is $R_{\text {in }}=48_{-20}^{+62} \mathrm{R}_{\mathrm{g}}$, and the inclination angle is $i=41_{-12}^{+29 \circ}$; while the EWs of the broad and narrow components are $\mathrm{EW}_{\text {Diskline }}=53_{-13}^{+14} \mathrm{eV}$ and $\mathrm{EW}_{\mathrm{N}}=64 \pm 6 \mathrm{eV}$ respectively. If the constraint on the disk emissivity is relaxed and a flatter emissivity is assumed $(q=2)$, then a disk inner radius of $6 R_{\mathrm{g}}$ is allowed by the present data. The ratio between the data and this best fit model is shown in Fig. 5 (lower panel), an absorption line near $8 \mathrm{keV}$ is the only residual. Adding an absorption line to our best fit model improved the fit $\left(\Delta \chi^{2}=20\right.$ for 2 additional parameters, $|E W| \sim 30 \mathrm{eV}, \mathrm{E} \sim 7.9 \mathrm{keV})$. In Fig. 7 the resulting EPICpn spectrum and the best model components are shown. The parameters derived for the diskline did not change significantly; the main difference is a slight reduction in its EW, which became now $46_{-13}^{+14} \mathrm{eV}$. Taking into account that this absorption feature is indicative of the presence of an ionized absorber (see section 4.1 and 5.3), we tested if the presence of a more complex absorber could mimic the profile of the detected broad component. We found that the inclusion of a two layers of absorption, characterized by a high $(\log \xi=3.7)$ and low $(\log \xi=2)$ ionization level, did not impact the detection of the broad component and its parameters. 


\section{Variability of the Iron line and continuum}

During the XMM-Newton observation, the 2-10 keV flux of MCG-5-23-16 varied from

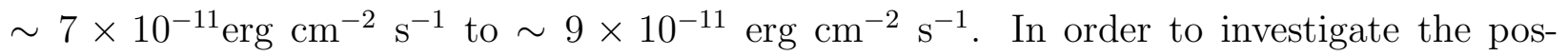
sible variability of the line properties and continuum shape, we first tested if there was any clear difference between the spectra extracted when the source was in relatively higher and lower flux states. We extracted spectra using $2-10 \mathrm{keV}$ threshold of $<4.6$ counts

$\mathrm{s}^{-1}\left(\mathrm{~F}_{(2-10 \mathrm{keV})} \lesssim 7.4 \times 10^{-11} \mathrm{erg} \mathrm{cm}^{-2} \mathrm{~s}^{-1}\right)$ and $>5.5$ countss $^{-1}\left(\mathrm{~F}_{(2-10 \mathrm{keV})} \gtrsim 8.8 \times 10^{-11}\right.$ erg $\left.\mathrm{cm}^{-2} \mathrm{~s}^{-1}\right)$. We then fitted both spectra with the previous best-fit model, replacing the diskline with a broad Gaussian. We found no evidence of variability of either the broad or the narrow component; indeed their normalizations are consistent within the errors (see Table 3). Furthermore, the width of the broad line is constant. In order to confirm that the line is not strongly variable, we checked the difference spectrum, obtained by subtracting the low from the high state data. The difference spectrum can be modeled with an absorbed power law with photon index $\Gamma=1.80 \pm 0.09$; no strong residuals are left in either the soft band or in the iron $\mathrm{K}$ band.

As a second test to assess the possible variability of the Fe emission complex, we divided the XMM-Newton observation into 5 intervals with a duration of $20 \mathrm{ksec}$ each; for the fit we tied all model parameters except the normalization of the primary power-law component. This model gives a statistically acceptable fit for all the 5 spectra. Fig. 8 shows the 5.5-8.5 $\mathrm{keV}$ residuals to this model for all five intervals; no strong deviations from the model are required at the energy of the iron $\mathrm{K} \alpha$ line. We then allowed the normalizations of the narrow and broad components free to vary. Fig. 9 (panel a and panel b) shows the fluxes of both the narrow and broad components. There is no evidence of variability during the present observation; furthermore, the fluxes of both components are consistent within the errors with the values measured in the previous XMM-Newton and Chandra observations (Dewangan et al. 2003; Balestra et al. 2004). This lack of variability can be explained by taking into account that MCG-5-23-16 is not highly variable on either short and relatively long time-scales; indeed the source has remained at a similar flux level $\left(7-9 \times 10^{-11} \mathrm{erg} \mathrm{cm}^{-2} \mathrm{~s}^{-1}\right)$ for the last 10 years.

The most striking feature that appears to be variable during the $X M M$-Newton observation is a possible absorption line at $\sim 7.7 \mathrm{keV}$ ( $\mathrm{E} \sim 7.66 \mathrm{keV}$, observer frame). This feature is present in the average EPIC pn spectrum but as shown in Fig. 8, it is strongest in the third spectrum (40 ksec after the beginning of the XMM-Newton observation). To illustrate this in Fig. 9 (panel c) we compare the intensity of this absorption feature during the five intervals. For this purpose we modeled the absorption with an inverted Gaussian line and fixed the energy to the best-fit value found with the spectral analysis of the third interval 
$\left(\mathrm{E} \sim 7.7 \mathrm{keV},|I| \sim 3.2 \times 10^{-5}\right.$ photons $\left.\mathrm{cm}^{-2} \mathrm{~s}^{-1}, \mathrm{EW}=52 \pm 15 \mathrm{eV}\right)$. The line is clearly variable and it appears to be strongest during the interval with the slightly higher $2-10 \mathrm{keV}$ flux (see Fig. 8, panel d), while it is barely detected in the other four spectra.

\subsection{A variable absorption feature at $7.7 \mathrm{keV}$}

\subsubsection{Epic-pn background and calibration checks.}

Before attempting any further modeling, we performed several tests to exclude that the $7.7 \mathrm{keV}$ absorption feature is due to inappropriate background, binning or pattern selection. The EPIC-pn background near this energy range presents two instrumental lines due to $\mathrm{Cu}$ $(8.05 \mathrm{keV})$ and $\mathrm{Ni}(7.48 \mathrm{keV}) \mathrm{K} \alpha$ emission lines and an inadequate background selection could in principle cause spurious absorption features. However several arguments exclude this possibility. First of all the net count rate $\left(\sim 2.56\right.$ counts $\left.\mathrm{s}^{-1}\right)$ of MCG-5-23-16 in the 5 -10 keV band is $\sim 300$ times greater than the background $\left(\sim 8 \times 10^{-3}\right.$ counts s$\left.^{-1}\right)$. Second, the sporadic nature of the feature is indicative that the feature can not be an artifact of the background or calibration of the EPIC-pn. Finally there was no background flaring activity during this time interval. We conclude that the feature is not due to instrumental or external background.

In order to exclude the possibility that the $7.7 \mathrm{keV}$ feature is due to a binning effect we rebinned the pn data of the third interval with a constant energy binning of $80 \mathrm{eV}$. This choice corresponds to about half of the energy resolution of the EPIC-pn camera in this energy range $(\mathrm{FWHM} \sim 150 \mathrm{eV}$ at $6.4 \mathrm{keV}$; see the XMM-Newton Users' Handbook, Ehle et al. 20065). As shown in Fig. 10, the residuals left by the time averaged best fit model confirm the presence of the feature, thus excluding the possibility that it is an artifact of the choice of the binning.

To exclude a pattern selection effect we then compared the pn spectra extracted with the pattern 0-4 and pattern 0 selection criteria. Though the latter has $30 \%$ fewer counts we found no significant difference in the absorption line parameters $\left(\Delta \chi^{2}=26\right.$ for 2 dof; $\mathrm{E} \sim 7.7$ $\mathrm{keV}, \mathrm{EW} \sim 60 \mathrm{eV})$. Finally the presence of an absorption feature is confirmed by the MOS1 and MOS2 spectra extracted in the same time interval. Although, due to the lower photon statistic, the significance of the absorption line is lower in these spectra, both the flux and the energy of the feature are consistent $\left(|I|=2.2 \pm 1.8 \times 10^{-5}\right.$ photons $\mathrm{cm}^{-2} \mathrm{~s}^{-1} \mathrm{E}=7.4 \pm 0.2 \mathrm{keV}$

\footnotetext{
${ }^{5}$ http://xmm.vilspa.esa.es/external/xmm_user_support/documentation/uhb/XMM_UHB.html
} 
$\Delta \chi^{2}=6$; see Table 4) with the values found with the pn data6. Furthermore the presence of the absorption feature is confirmed by the simultaneous Suzaku observation, indeed a weak absorption feature is present in the time averaged spectrum (see panel c of Fig. 6 in Reeves et al. 2007). The absorption line is weaker than in the XMM-Newton observation, which could be explained if we take into account the apparent sporadic nature of the feature, with a dilution effect due to the longer duration time $(\sim 220 \mathrm{ksec})$ of the Suzaku observation in the Earth orbit. Although the absorption line is not well constrained in the time-averaged Suzaku spectrum, the energy of the line at $7.8 \pm 0.1 \mathrm{keV}$ is coincident with the XMM-Newton data, while the flux of the line is weaker $|I|=1.8 \pm 0.9 \times 10^{-5}$ photons $\mathrm{cm}^{-2} \mathrm{~s}^{-1}$ (see Table 4).

\subsubsection{Modeling the absorption feature of the third segment.}

We first fitted the absorption feature adding a Gaussian shaped absorption line keeping its width fixed to $\sigma=0.1 \mathrm{keV}$. The addition of this line improved the fit with a $\Delta \chi^{2}=33$ for 2 additional parameters $\left(\chi^{2} / d o f=1451 / 1505\right)$. The line energy is $7.72 \pm 0.06 \mathrm{keV}$ with a $|\mathrm{EW}|$ of $52 \pm 15 \mathrm{eV}\left(|I|=3.2 \pm 0.9 \times 10^{-5}\right.$ photons $\left.\mathrm{cm}^{-2} \mathrm{~s}^{-1}\right)$. Leaving the width of the line free did not improve the fit significantly $\left(\Delta \chi^{2}=5\right.$ for one additional parameter $)$. With this fit we find $\sigma=0.2 \pm 0.1 \mathrm{keV}, \mathrm{EW}=78 \pm 29 \mathrm{eV}$ and an energy consistent with the previous best fit $(\mathrm{E}=7.71 \pm 0.08 \mathrm{keV})$. In Fig. 11 we show the confidence contour plot of the line parameters (rest frame energy and intensity) with the line width left free to vary. We also attempted to fit the absorption feature replacing the Gaussian line with an edge due to K-shell absorption from partially ionized iron. This model gives a best-fit energy of $7.33_{-0.22}^{+0.12}$ $\mathrm{keV}$ and an optical depth of $\tau=0.09 \pm 0.03$. The fit is statistically acceptable; however, it is worse than the Gaussian absorption model $\left(\chi^{2} / d o f=1465 / 1505\right.$, which correspond to a $\Delta \chi^{2}=14$ worse compared to the absorption line) and more importantly, it is unsuccessful at modeling the residuals at $7.7 \mathrm{keV}$.

\footnotetext{
${ }^{6}$ Unfortunately the Chandra observations do not overlap with this segment of the XMM-Newton spectrum. However two possible weak absorption features appear to be present at the rest frame energy of about $7.3 \mathrm{keV}$ and $7.4 \mathrm{keV}$ (see Fig. 6) suggesting possible variability of the absorber, although the statistical significance of these features is low.
} 


\subsubsection{The significance of the detection of the absorption line}

By applying the standard two-parameter $F$-test to the drop in $\chi^{2}$ of 33 for the addition of an inverted Gaussian at $7.71 \mathrm{keV}$, we found a null hypothesis probability for adding this extra component of $\sim 4 \times 10^{-8}$. However, as discussed by Protassov et al. (2002), the $F$-test applied in this way could overestimate the true significance of the detected absorption line. In particular the $F$-test does not take into account the number of time bins in which the line is searched as well as the range of energy where the line might be expected (see Porquet et al. 2004).

To asses the significance of the detection we then performed Monte Carlo simulations as described in Porquet et al. (2004) and in Markowitz et al. (2006), for a similar case of a blue-shifted Fe K $\alpha$ absorption line detected in the XMM-Newton observation of IC 4329a. We assumed as our null hypothesis model the best fit model with no absorption feature, and we simulated 1000 spectra with the photon statistics expected for a $20 \mathrm{ksec}$ exposure. Each simulated spectrum was then fitted with the null hypothesis model to obtain a $\chi^{2}$ value and we systematically searched for an absorption line over the 4-9 keV energy range stepping the energy centroid of the Gaussian in increments of $0.1 \mathrm{keV}$, refitting at each step. We then obtained for each simulated spectrum a minimum $\chi^{2}$ and created a distribution of 1000 simulated values of the $\Delta \chi^{2}$ (compared to the null hypothesis model), which was used to construct a cumulative frequency distribution of the $\Delta \chi^{2}$ expected for a blind line search in the 4-9 keV range. Not a single fake spectrum had a $\left|\Delta \chi^{2}\right| \geq 33$, thus the inferred probability that the null hypothesis model was correct is $<0.1 \%$. Taking into account the number of intervals (5) into which the observation had been split, we derive that the line detection is significant at $>99.5 \%$.

Finally, we performed a similar Monte Carlo simulation to test the statistical significance of the absorption feature in the MOS spectra. For simplicity we ran the simulation on MOS1 only and we found that in this case the significance is only $\sim 61 \%$, mainly due to the lower $\mathrm{S} / \mathrm{N}$ of the MOS spectra at this energy. However the fact that the absorption feature is detected by the pn, both MOS cameras and Suzaku suggests that the feature is likely real and not an artefact.

\subsubsection{The ionized absorber model.}

As already discussed from X-ray spectroscopic observation of several other Seyfert galaxies (i.e. MCG-6-30-15, Young et al. 2005; NGC 3783, Reeves et al. 2004; Mrk 509, 
Dadina et al. 2005; Mrk 335 Longinotti et al. 2007; E1821+643, Yaqoob \& Serlemitsos 2005; IC 4329a, Markowitz et al. 2006) and QSOs (i.e. PG 1211+143, Pounds et al. 2003; PDS 456, Reeves et al. 2003; APM 08279+5255, Chartas et al. 2002; PG 1115+080, Chartas et al. 2003) a likely candidate for the $7.7 \mathrm{keV}$ feature is blue-shifted K-shell absorption due to Heor H-like iron. In particular if we assume that the line is due to H-like iron (Fe xxvi Ly $\alpha$ at $6.97 \mathrm{keV}$ ) the observed blue-shift suggests that the absorber is outflowing with a velocity of the order of $0.1 c$.

In order to obtain a more physical representation of the absorber, we replaced the Gaussian line with a model comprised of a grid of photoionized absorbers generated by the XSTAR photoionization code (Bautista \& Kallman 2001). For the absorber we assumed a one zone photoionization model with half solar abundances and a turbulence velocity of $1000 \mathrm{~km} \mathrm{~s}^{-1}$. The free parameters of this model are: the column density $\left(N_{\mathrm{H}}\right)$, the outflowing velocity of the absorber $\left(v_{\text {out }}\right)$ and the ionization parameter $\left(\xi=L / n r^{2}\right.$; where $L$ is the ionizing luminosity, $n$ is the electron density, and $r$ the absorber distance). To reproduce the absorption feature a column density of $\sim 8 \times 10^{22} \mathrm{~cm}^{-2}$ and an ionization state characterized by $\log \xi=3.7_{-0.3}^{+0.2} \mathrm{erg}_{\mathrm{cm} \mathrm{s}}^{-1}$ are required with an outflow velocity of $0.09 \pm 0.01 c(\sim 30000$ $\mathrm{km} \mathrm{s}^{-1}$ ). A plot of this best-fit model is shown in Fig. 12 (model A). The column density is not well constrained and we can place only a lower limit of $>2 \times 10^{22} \mathrm{~cm}^{-2}$. At this ionization level, the Fe K-shell absorption is indeed mainly due to Fe XXVI and is consistent with absorption from highly ionized iron outflowing at $\sim 0.1 c$ with respect to systemic.

A lower velocity outflow could in principle be obtained assuming that the feature is due to a Fe XXIV 1s-3p line at $7.78 \mathrm{keV}$. This is illustrated in Figure 11 (model B), which illustrates an XSTAR model with a column density of $N_{\mathrm{H}}=10^{23} \mathrm{~cm}^{-2}$, an ionization param-

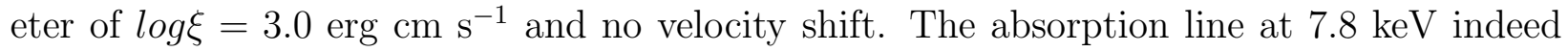
corresponds to the above FeXXIV $1 \rightarrow 3$ transition. However, in this ionization regime $(\log \xi \sim 2.5-3.0)$, we would expect to detect absorption trough at $6.5-6.7 \mathrm{keV}$ due to a blend of the $1 \rightarrow 2$ transitions of Fe XVIII-XXV; however, this is not observed in the MCG 5-23-16 spectrum. In particular, at this lower ionization we would expect to see the strong absorption due to the Fe XXIV 1s-2p absorption line at $6.67 \mathrm{keV}$, and for $\log \xi=3.0$ erg $\mathrm{cm} \mathrm{s}^{-1}$ (as illustrated in the figure) we would also expect to see an even deeper absorption feature at $6.7 \mathrm{keV}$ due to the Fe XXV resonant absorption. Furthermore in this ionization regime $(\log \xi \sim 2.5-3.0)$, several strong absorption lines from iron L-shell $(2 \rightarrow 3)$ transitions as well as $\mathrm{He}$ and $\mathrm{H}$-like $\mathrm{Si} / \mathrm{S} \mathrm{K}$-shell lines are expected near $1-2 \mathrm{keV}$, which are not observed in either the XMM-Newton Suzaku, or Chandra HETG spectra. Therefore this lower velocity solution appears to be ruled out. 
Finally it is possible to have a low ionization $\mathrm{K} \beta$ absorption line without strong $\mathrm{K} \alpha$ absorption from the same species. This scenario is shown in Figure 11 (model C) for an absorber with a column density of $N_{\mathrm{H}}=10^{23} \mathrm{~cm}^{-2}$ and an ionization parameter of $\log \xi=1.5$ erg $\mathrm{cm} \mathrm{s}^{-1}$. Indeed, at this ionization state the dominant species is iron less ionized then Fe XVII; the L-shell is full and cannot produce the $1 s-2 p$ absorption between $6.4-7.0 \mathrm{keV}$. However, there is an absorption line from $7.1-7.2 \mathrm{keV}$ due to $1 \rightarrow 3$ transitions from Fe less ionized then Fe XVII. In this scenario a blueshift of $\sim 0.08 c$ would still be required to model the absorption line at $7.7 \mathrm{keV}$ in the spectrum. Furthermore the column density required to model the EW of the $\mathrm{K} \beta$ absorption feature is $N_{\mathrm{H}}>>10^{23} \mathrm{~cm}^{-2}$, which would introduce too much continuum (bound-free) absorption below $6 \mathrm{keV}$, inconsistent with the pn data.

Therefore we conclude that the fast $(0.1 c)$ high ionization outflow is the most likely model to account for the absorption feature at $7.7 \mathrm{keV}$. Moreover, when we compare the $2-10 \mathrm{keV}$ continuum and the neutral $N_{\mathrm{H}}$ measured during the third interval with the other intervals and with the average spectrum, we do not find any statistically significant difference $\left(\Delta N_{H}<10^{21} \mathrm{~cm}^{-2}\right)$, which rules out the possible presence of variable neutral and/or low ionization absorber.

\section{Discussion and Conclusions}

We have presented the results from XMM-Newton and Chandra observations of MCG-523-16, which are part of a simultaneous campaign conducted in December 2005 also comprising Suzaku and RXTE observations. The 0.5-10 keV continuum of MCG-5-23-16 resembles at first order the canonical X-ray emission expected from a Compton thin Seyfert 2 galaxy: an absorbed $\left(N_{\mathrm{H}}=1.5 \times 10^{22} \mathrm{~cm}^{-2}\right)$ power law component $(\Gamma=1.82)$, which emerges at energy $\gtrsim 1 \mathrm{keV}$, and a steep soft excess, which is well fitted by a power law component plus several emission lines from $\mathrm{O}, \mathrm{Ne}$, and $\mathrm{N}$. The XMM-Newton observation of MCG-5-23-16 confirms the presence of the Fe $\mathrm{K} \alpha$ emission complex, which is well described by a narrow $\mathrm{Fe}$ $\mathrm{K} \alpha$ emission line superimposed on a relativistically broadened component. The simultaneous Suzaku observation provided us with an accurate description of the underlying continuum which allowed us to perform detailed modeling of the Fe K emission line complex. Finally due to the sufficiently long duration, the XMM-Newton RGS spectra have enough photon statistics to investigate the origin of the soft X-ray emission. 


\subsection{The origin of the soft X-ray emission}

The analysis of the EPIC-pn and MOS spectra of MCG-5-23-16 revealed the presence, below $1 \mathrm{keV}$, of a soft excess with respect to the primary nuclear emission. This soft excess can be well fitted by adding an un-absorbed power law component to the primary AGN emission, the photon index of this power law is found to be steeper $(\Gamma \sim 3.1)$ than the primary AGN component $(\Gamma \sim 1.8)$, and even at the EPIC CCD resolution an emission line is detected around $0.9 \mathrm{keV}$.

A soft excess below $1 \mathrm{keV}$ is not unusual in obscured Seyfert galaxies like MCG-5-23-16 (see Bianchi et al. 2006 and references therein) and it has been already suggested that it could be due to a superimposition of scattered emission into the line of sight by ionized gas plus several emission lines from highly ionized ( $\mathrm{He}$ and $\mathrm{H}$ like) elements (i.e Mrk 3: Sako et al. 2000; Pounds \& Page 2005; Bianchi et al. 2005; Circinus: Sambruna et al. 2001; NGC 1068: Kinkhabwala et al. 2002; Ogle et al. 2003; Brinkman et al. 2002; NGC 4507: Matt et al. 2004). Key diagnostics to understand the origin of this X-ray emission when a high resolution spectrum is available are: the detection of $\mathrm{RRC}$ transitions, the detection of enhanced K-shell emission lines from H-like and He-like ions, the detection of Fe L-shell emission and the ratio between the forbidden and the recombination transition in the He-like triplets.

This long XMM-Newton observation has provided for the first time RGS spectra for MCG-5-23-16 with sufficient photon statistics to perform a detailed modeling of the soft X-ray emission, allowing for the first time in this object the detection of the O vII RRC. The width of this emission line indicates that the recombining electron temperature is a few $\mathrm{eV}\left(k_{\mathrm{B}} T<24 \mathrm{eV}\right)$. This is suggestive that the emitting plasma is photoionized rather then

collisionally ionized (Liedahl \& Paerels 1996). This is also indicative that the soft X-ray emission is probably dominated by scattering of the primary AGN emission rather than due to emission from hot gas in the host galaxy, e.g. from starburst activity. Our analysis of the RGS spectra of MCG-5-23-16 confirms the detection of the O VII and O vIII lines previously reported by Guainazzi \& Bianchi (2007) and the line fluxes are in agreement with the measurement obtained with the analysis of these previous short XMM-Newton observations of MCG-5-23-16. We cannot exclude on a statistical ground the possible presence of emission due to a collisionally ionized plasma. Indeed the spectra can be equally modeled by replacing these emission lines with a multi temperature thermal model; which represents the emission due to a collisionally ionized plasma. However the "AGN" model (scattered power law component plus several photoionized emission lines) is preferred because of the low ion abundance obtained $\left(Z<0.2 Z_{\odot}\right)$ in the thermal model, which reflects the lack of a strong Fe L-shell emission with respect to the fluxes of the Oxygen lines. 
We therefore conclude that the most likely the origin of the soft X-ray emission is due to a plasma photoionized by the AGN. This plasma must be located outside the Compton-thin absorber and, as already suggested for other Seyfert 2 galaxies, it could be coincident with the NLR.

\subsection{The Fe $\mathbf{K} \alpha$ emission complex}

This deep XMM-Newton observation of MCG-5-23-16 confirms the presence of broad and narrow iron $\mathrm{K} \alpha$ emission lines, which were reported since the first $A S C A$ observation (Weaver et al. 1997). The ChandraHETG spectrum clearly reveals a narrow line at $E_{N}=$ $6.40 \pm 0.02 \mathrm{keV}$ with a FWHM $<5000 \mathrm{~km} \mathrm{~s}^{-1}$ and a flux of $5.6 \pm 0.7 \times 10^{-5}$ photons $\mathrm{cm}^{-2}$ $\mathrm{s}^{-1}$. The intensity of this component is found to be constant, within the errors, during this observation and also when comparing with previous observation $\left(<I_{N}\right\rangle=4.5 \times 10^{-5}$ photons $\mathrm{cm}^{-2} \mathrm{~s}^{-1}$, Balestra et al. 2004; $I_{N}=6.5 \pm 2.7 \times 10^{-5}$ photons $\mathrm{cm}^{-2} \mathrm{~s}^{-1}$, Weaver et al. 1997). The constancy in flux of this line together with the limits on the width obtained with Chandra are suggestive of an origin from distant matter such as the putative torus. Indeed the upper limit on the FWHM corresponds to a distance from the central black hole greater then $10^{4} \mathrm{R}_{g}$.

The presence of two Compton-thick X-ray reprocessors responsible for the two components of the iron line, suggested since the $A S C A$ observation (Weaver et al. 1997) is confirmed with this deep XMM-Newton observation and with the deep Suzaku observation (Reeves et al. 2007). The geometry inferred for MCG-5-23-16 with this latter observation is discussed in detail in Reeves et al. (2007). To summarize, one plausible scenario is that we are seeing $\mathrm{MCG}-5-23-16$ through the Compton thin edge of the putative torus, which is Compton thick at the plane of the accretion disk. This is in agreement with the inclination $\left(i=41_{-12}^{+29}\right)^{7}$ of the accretion disk derived by modeling the broad line component with a relativistic line profile. The column density of $1.5 \times 10^{22} \mathrm{~cm}^{-2}$ measured using low energy cut-off is thus associated with the thinner absorbing material, e.g. encountered viewing through the edge of the torus. We found no evidence of variability of the column density of this absorber within this long observation, and also no strong variability is found when comparing the column densities measured with the previous observations performed with BeppoSAX

\footnotetext{
${ }^{7}$ Modeling the combined Suzaku and XMM-Newton spectra, Reeves et al. (2007) found an inclination of $i=53_{-9}^{+7} \circ$ which is in agreement within the errors with the value obtained from the analysis of the $X M M-N e w t o n$ spectrum.
} 
(Risaliti 2002a), ASCA (Weaver et al. 1997), XMM-Newton and Chandra (Dewangan et al. 2003; Balestra et al. 2004). This result implies that the absorber is probably far from the central black hole and there is no evidence that this absorber is clumpy as suggested for other Seyfert 2s (Risaliti et al. 2002b).

This deep XMM-Newton observation confirms the presence of a relativistically broadened iron $\mathrm{K} \alpha$ line; the width derived from modeling this component with a Gaussian profile corresponds to a FWHM $\sim 40000 \mathrm{~km} \mathrm{~s}^{-1}$ and is suggestive of an origin in the accretion disk. The profile of this component is nearly symmetric and can be modeled, equally well with a broad Gaussian or a relativistic profile; in the latter case the derived inner radius is about 20-40R $R_{g}$. Since the advent of XMM-Newton and Chandra one of the most debated issues in the study of the broad iron $\mathrm{K} \alpha$ lines has been the fraction of AGNs which clearly show the presence of a broad component (Nandra et al. 2006; Guainazzi et al. 2006). Several authors have discussed the robustness of the detection in some objects of a broad iron line (i.e. NGC 3516, Turner et al. 2005; NGC 3783, Reeves et al. 2004). This controversy emerged when observations characterized by high photon statistics showed the ambiguity of modeling the iron $\mathrm{K} \alpha$ line when complex absorption is present (Pounds et al. 2003, 2004). Indeed, a high column density warm absorber can produce curvature in the spectrum at the energy of the iron $\mathrm{K} \alpha$ line that mimics the profile of a relativistically broadened emission line. The detection of the absorption feature at $\sim 7.8 \mathrm{keV}$ shows that a high column density variable absorber (a high velocity, highly ionized outflow) is also present in MCG-5-23-16; this could in principle give rise to ambiguity in the interpretation of the broad component. However, in the case of MCG-5-23-16 the availability of a simultaneous observation with Suzaku above $10 \mathrm{keV}$ allowed us to tightly constrain the underlying X-ray continuum and to rule out the interpretation of the broad line as due to unmodeled complex absorption. Indeed the residuals left at the energy of the iron $\mathrm{K} \alpha$, when we take into account the amount of reflection detected with Suzaku, cannot be explained by the effect of complex absorption. Furthermore, once the absorber responsible for the feature detected at $\sim 7.8 \mathrm{keV}$ is accounted for, either in the time-averaged spectrum or in the third segment of the XMM-Newton observation, a broad line is still required by the data with a similar EW and FWHM. Note that the ionization parameter of the absorber is required to be high and does not introduce additional spectral curvature below $6 \mathrm{keV}$, which hence does not impact the broad iron line modeling.

The remaining open questions on the origin of the broad line in MCG-5-23-16 are the relatively large inner radius derived for the accretion disk, and its lack of variability. The former can be explained with several scenarios: the disk could be truncated or missing below $20 \mathrm{R}_{g}$, or the inner part of the accretion disk could be so highly ionized that the iron is fully ionized. However it worth noting that, as shown by Reeves et al. (2007), assuming a flat emissivity $(q=2)$ a inner radius of about $6 \mathrm{R}_{g}$ (in the case of a Schwarzschild black hole) 
cannot be statistically ruled out.

The second open issue is the lack of variability of the iron emission line both on short and long time-scales. Indeed the flux of the broad component is found to be consistent with being constant when using the short-term time-resolved spectroscopy performed within this deep observation, and when comparing our result with the long-term flux history presented in Balestra et al. (2004). The strength of the broad component appears to be lower during this observation with respect to the value reported since the first $A S C A$ observation $(\mathrm{EW} \sim 200$ $\mathrm{eV}$; Weaver et al. 1997), however when we take into account the larger errors on the early $A S C A$ measurements we cannot exclude the line being constant.

In MCG-5-23-16 this lack of variability of the iron emission line is not so striking as in MCG6-30-15 (Miniutti et al. 2007; Vaughan \& Fabian 2004), due to the low level of variability of the intrinsic continuum (30-40\% compared to a factor of 2-3 in case of MCG-6-30-15).

\subsection{The blue-shifted absorption line: a possible high velocity, ionized variable outflow}

Perhaps the most interesting result of this long XMM-Newton observation has been the discovery of a possibly variable absorption line from ionized iron. The feature appears to be transient with a time scale of about $20 \mathrm{ksec}$ and it is detected at an observed energy of about $7.66 \mathrm{keV}$ (corresponding to a rest-frame energy of $7.72 \mathrm{keV}$ ). As shown the most plausible association of this feature is with K-shell absorption from H-like iron, which is blueshifted by $\sim 0.1 c$. Indeed modeling this absorption feature with XSTAR (Bautista \& Kallman 2001) requires a column density of about $8 \times 10^{22} \mathrm{~cm}^{-2}$ and a high ionization parameter $\left(\log \xi=3.7 \pm 0.3 \mathrm{erg}^{\left.\mathrm{cm} \mathrm{s}^{-1}\right)}\right.$ which implies that the absorption is due to a blueshifted $1 \rightarrow$ 2 transition of Fexxvi $(E=6.97 \mathrm{keV})$. The velocity of the absorbing material is found to be $v=(0.09 \pm 0.01) c$.

In the last few years, red- and blue-shifted absorption lines associated with the presence of highly ionized gas in- and/or out-flowing at relativistic velocities have been reported for several AGN (E1821+643 Yaqoob \& Serlemitsos 2005; Mkn 509 Dadina et al. 2005; NGC 3516 Nandra et al. 1999; Turner et al. 2005; Mrk 335, Longinotti et al. 2007). These absorption lines are found both in Seyfert galaxies (NGC 3783, Reeves et al. 2004; IC4392a, Markowitz et al. 2006; NGC 1365, Risaliti et al. 2005; Ark 564, Papadakis et al. 2007) as well as in quasars (PG 1211+143, Pounds et al. 2003; PDS 456, Reeves et al. 2003) and BAL QSOs (APM 08279+5255, Chartas et al. 2002; PG 1115+080, Chartas et al. 2003). These absorption systems can also be variable on different time scales, in their ionization state and column density, with the most extreme cases being NGC 1365 (Risaliti et al. 2005) 
and Mkn 509 (Dadina et al. 2005). From the analysis of the different intervals in which we split the observation we can infer that it is unlikely that the variability of the absorber in MCG-5-23-16 is due to a change in the ionization state of the outflowing material, otherwise we would detect signatures of this absorber in all the spectral slices. A more likely scenario is a change in column density of this absorber.

The present data suggest we are seeing a transient absorber, which could be associated with a cloud which sporadically obscures the central source. This "cloud" could be the signature of a clumpy absorber located close to the central X-ray source or of matter ejected sporadically. Different models have been proposed to explain the powerful outflows detected with the recent XMM-Newton and Chandra observations; in particular transient red and blue shifted absorption lines are predicted in several theoretical models of failed disk winds (Proga et al. 2000; King \& Pounds 2003) or an aborted jet (Ghisellini et al. 2004). The picture emerging is that these features can provide a direct probe of the dynamics and kinematics of the innermost central regions of AGNs. For the absorber detected in MCG-523-16 the data suggest that this feature appears when the source reaches a relative maximum in the intrinsic 2-10 keV flux. However, monitoring the spectrum on longer timescales would be required to determine whether there is a statistically firm correlation between the presence of the absorber and the source brightness and determine if any duty cicle is present. Thus both a scenario where a clumpy absorber or a variable or failed outflow or jet are at present possible. Finally, it is worth noticing that the rapid variability of this absorption feature is indicative of a compact absorber and rules out a possible $z=0$ origin, e.g. due to the warm intergalactic medium (WHIM) or a local hot bubble as claimed along the line of sight to other AGN (McKernan et al. 2004, 2005).

Before deriving an estimate of the location, mass and energetics associated with the absorber we performed a consistency check for the $N_{\mathrm{H}}$ value measured with the XSTAR model and the EW $(\sim 50 \mathrm{eV})$ of the absorption line measured with the Gaussian component. Following the curve of growth for H-like iron (see Fig. 4 of Risaliti et al. 2005) we inferred that the detected EW requires a turbulence velocity greater than $500 \mathrm{~km} \mathrm{a}^{-1}$; a lower turbulence velocity would imply a Compton thick absorber and a similar EW of the H-like $\mathrm{K} \beta$ line (due to saturation of the $\mathrm{K} \alpha$ line), which is not detected $(|E W|<15 \mathrm{eV}$ at the $90 \%$ confidence level). On the other hand a turbulence velocity greater than $3000 \mathrm{~km} \mathrm{~s}^{-1}$ would produce a broad absorption feature which would be resolved even at the EPIC-pn resolution at 8 $\mathrm{keV}(\sim 170 \mathrm{eV})$. We therefore conclude that the observed EW and line width are in broad agreement with the XSTAR estimate of a column density of about $10^{23} \mathrm{~cm}^{-2}$, for a turbulence velocity $\sigma=1000 \mathrm{~km} \mathrm{~s}^{-1}$. Using this value for the column density we can now estimate the maximum distance of this cloud or blob from the central black hole using the relation be- 
tween the ionization parameter, the density of the absorber and the illuminating continuum luminosity: $L / \xi=n R^{2}$; where $L$ is the intrinsic $2-100 \mathrm{keV}$ X-ray luminosity $\left(5.4 \times 10^{43} \mathrm{erg}\right.$ $\left.\mathrm{s}^{-1}\right)$. Assuming then that the thickness of the cloud $\Delta R=N_{H} / n$ is less than the distance $\mathrm{R}$, we find $R<10^{17} \mathrm{~cm}$. A lower limit for the distance of the absorber, assuming it is in the form of an outflow, can be obtained equating the velocity of the absorbing material to the escape velocity at a given radius $\mathrm{R}$ from the central black hole; the derived distance is then $R \gtrsim 100 R_{g}$. A constraint on the size of this cloud can be placed assuming $20 \mathrm{ksec}$ as the characteristic variability timescale, when our line of sight intercepts the absorbing cloud; this gives us $\Delta R \sim 6 \times 10^{13} \mathrm{~cm}$, which corresponds to $\sim 10 R_{g}$ for a black hole mass of $5 \times 10^{7} M_{\odot}$ (Wandel \& Mushotzky 1986). We can then infer a density of $\sim 10^{9} \mathrm{~cm}^{-3}$ and assuming a spherical geometry for the cloud, a mass of about $10^{28} \mathrm{~g}$. These one order of magnitude estimates for the mass and velocity correspond to a kinetic energy $E_{\text {kin }} \sim 5 \times 10^{46} \mathrm{erg}$ and using the $20 \mathrm{ksec}$ as a characteristic timescale to a power of $\sim 2.5 \times 10^{42} \mathrm{erg} \mathrm{s}^{-1}$. This value corresponds to $\sim 10 \%$ of the $2-10 \mathrm{keV}$ X-ray luminosity and is thus is agreement with a radiation driven wind model (Proga \& Kallman 2004) or with an aborted jet (Ghisellini et al. 2004).

In conclusion, this deep XMM-Newton observation revealed that the soft X-ray emission of MCG-5-23-16 can be ascribed to material photoionized by the AGN, likely to be located outside the sub-pc scale of the absorber and perhaps coincident with the NLR. We confirm the presence of a iron $\mathrm{K} \alpha$ emission line complex composed by a narrow and a broad relativistic component. The inclination derived from the diskline profile $\left(i \sim 40^{\circ}\right)$ is in agreement with the orientationally based Unification Scheme of AGN (Antonucci 1993), the X-ray classification of MCG-5-23-16 as Compton thin Seyfert 2 (i.e. intermediate between a type 1 AGN and a Compton thick type 2) and the optical classification as a Seyfert 1.9. Finally we detected a sporadic Fe K absorption feature which could be a signature of a variable high velocity outflow. This detection adds one more example to the increasing sample of AGN where relativistic outflows have been revealed in the X-ray band. The growing evidence of high velocity outflows in AGN indicates that they may play an important role in the energetics of AGN central engines.

We would like to thank the anonymous referee for his/her useful comments that have improved this paper. This paper has made use of observations obtained with XMM-Newton, an ESA science mission with instruments and contributions directly funded by ESA Member States and the USA (NASA). Support for this work was provided by the National Aeronautics and Space Administration through Chandra Award Number GO5-6146Z issued by the Chandra X-ray Observatory Center, which is operated by the Smithsonian Astrophysi- 
cal Observatory for and on behalf of the National Aeronautics Space Administration under contract NAS8-03060.

\section{REFERENCES}

Antonucci, R. 1993, ARA\&A, 31, 473

Balestra, I., Bianchi, S., \& Matt, G. 2004, A\&A, 415, 437

Bautista, M. A., \& Kallman, T. R. 2001, ApJS, 134, 139

Bianchi, S., Matt, G., Balestra, I., Guainazzi, M., \& Perola, G. C. 2004, A\&A, 422, 65

Bianchi, S., Miniutti, G., Fabian, A. C., \& Iwasawa, K. 2005, MNRAS, 360, 380

Bianchi, S., Guainazzi, M., \& Chiaberge, M. 2006, A\&A, 448, 499

Brinkman, A. C., Kaastra, J. S., van der Meer, R. L. J., Kinkhabwala, A., Behar, E., Kahn, S. M., Paerels, F. B. S., \& Sako, M. 2002, A\&A, 396, 761

Cappi, M. 2006, Astronomische Nachrichten, 327, 1012

Chartas, G., Brandt, W. N., Gallagher, S. C., \& Garmire, G. P. 2002, ApJ, 579, 169

Chartas, G., Brandt, W. N., \& Gallagher, S. C. 2003, ApJ, 595, 85

Cash, W. 1979, ApJ, 228, 939

Dadina, M., Cappi, M., Malaguti, G., Ponti, G., \& de Rosa, A. 2005, A\&A, 442, 461

den Herder, J. W., et al. 2001, A\&A, 365, L7

Dewangan, G. C., Griffiths, R. E., \& Schurch, N. J. 2003, ApJ, 592, 52

Dickey, J. M., \& Lockman, F. J. 1990, ARA\&A, 28, 215

Fabian, A. C., Rees, M. J., Stella, L., \& White, N. E. 1989, MNRAS, 238, 729

Fabian, A. C., Iwasawa, K., Reynolds, C. S., \& Young, A. J. 2000, PASP, 112, 1145

Ferruit, P., Wilson, A. S., \& Mulchaey, J. 2000, ApJS, 128, 139

Ghisellini, G., Haardt, F., \& Matt, G. 2004, A\&A, 413, 535

Guainazzi, M., Bianchi, S., \& Dovčiak, M. 2006, Astronomische Nachrichten, 327, 1032 
Guainazzi, M., \& Bianchi, S. 2007, MNRAS, 374, 1290

Kallman, T. R., Liedahl, D., Osterheld, A., Goldstein, W., \& Kahn, S. 1996, ApJ, 465, 994

King, A. R., \& Pounds, K. A. 2003, MNRAS, 345, 657

Kinkhabwala, A., et al. 2002, ApJ, 575, 732

Iwasawa, K., Wilson, A. S., Fabian, A. C., \& Young, A. J. 2003, MNRAS, 345, 369

Liedahl, D. A. 1999, LNP Vol. 520: X-Ray Spectroscopy in Astrophysics, 520, 189

Liedahl, D. A., \& Paerels, F. 1996, ApJ, 468, L33

Longinotti, A. L., Sim, S. A., Nandra, K., \& Cappi, M. 2007, MNRAS, 374, 237

Magdziarz, P., \& Zdziarski, A. A. 1995, MNRAS, 273, 837

Markert, T. H., Canizares, C. R., Dewey, D., McGuirk, M., Pak, C. S., \& Schattenburg, M. L. 1994, Proc. SPIE, 2280, 168

Markowitz, A., Reeves, J. N., \& Braito, V. 2006, ApJ, 646, 783

Matt, G. 2002, MNRAS, 337, 147

Matt, G., Bianchi, S., D’Ammando, F., \& Martocchia, A. 2004, A\&A, 421, 473

Mattson, B. J., \& Weaver, K. A. 2004, ApJ, 601, 771

McKernan, B., Yaqoob, T., \& Reynolds, C. S. 2005, MNRAS, 361, 1337

McKernan, B., Yaqoob, T., \& Reynolds, C. S. 2004, ApJ, 617, 232

Mewe, R., Gronenschild, E. H. B. M., \& van den Oord, G. H. J. 1985, A\&AS, 62, 197

Miniutti, G., et al. 2007, PASJ, 59, 315

Nandra, K., George, I. M., Mushotzky, R. F., Turner, T. J., \& Yaqoob, T. 1997, ApJ, 477, 602

Nandra, K., George, I. M., Mushotzky, R. F., Turner, T. J., \& Yaqoob, T. 1999, ApJ, 523, L17

Nandra, K., O’Neill, P. M., George, I. M., Reeves, J. N., \& Turner, T. J. 2006, Astronomische Nachrich 
Perola, G. C., Matt, G., Cappi, M., Fiore, F., Guainazzi, M., Maraschi, L., Petrucci, P. O., \& Piro, L. 2002, A\&A, 389, 802

Ogle, P. M., Brookings, T., Canizares, C. R., Lee, J. C., \& Marshall, H. L. 2003, A\&A, 402, 849

Porquet, D., \& Dubau, J. 2000, A\&AS, 143, 495

Porquet, D., Reeves, J. N., Uttley, P., \& Turner, T. J. 2004, A\&A, 427, 101

Protassov, R., van Dyk, D. A., Connors, A., Kashyap, V. L., \& Siemiginowska, A. 2002, ApJ, 571, 545

Papadakis, I. E., Brinkmann, W., Page, M. J., Mc Hardy, I., \& Uttley, P. 2007, A\&A, 461, 931

Pounds, K. A., Reeves, J. N., King, A. R., \& Page, K. L. 2004, MNRAS, 350, 10

Pounds, K. A., Reeves, J. N., King, A. R., Page, K. L., O’Brien, P. T., \& Turner, M. J. L. 2003, MNRAS, 345, 705

Pounds, K. A., \& Page, K. L. 2005, MNRAS, 360, 1123

Proga, D., \& Kallman, T. R. 2004, ApJ, 616, 688

Proga, D., Stone, J. M., \& Kallman, T. R. 2000, ApJ, 543, 686

Reynolds, C. S., \& Nowak, M. A. 2003, Phys. Rep., 377, 389

Reeves, J. N., O’Brien, P. T., \& Ward, M. J. 2003, ApJ, 593, L65

Reeves, J. N., Nandra, K., George, I. M., Pounds, K. A., Turner, T. J., \& Yaqoob, T. 2004, ApJ, 602, 648

Reeves, J. N., et al. 2007, PASJ, 59, 301

Risaliti, G. 2002, A\&A, 386, 379

Risaliti, G., Elvis, M., \& Nicastro, F. 2002, ApJ, 571, 234

Risaliti, G., Bianchi, S., Matt, G., Baldi, A., Elvis, M., Fabbiano, G., \& Zezas, A. 2005, ApJ, 630, L129

Sako, M., Kahn, S. M., Paerels, F., \& Liedahl, D. A. 2000, ApJ, 543, L115 
Sambruna, R. M., Netzer, H., Kaspi, S., Brandt, W. N., Chartas, G., Garmire, G. P., Nousek, J. A., \& Weaver, K. A. 2001, ApJ, 546, L13

Schurch, N. J., Warwick, R. S., Griffiths, R. E., \& Sembay, S. 2003, MNRAS, 345, 423

Tanaka, Y., et al. 1995, Nature, 375, 659

Turner, T. J., Kraemer, S. B., George, I. M., Reeves, J. N., \& Bottorff, M. C. 2005, ApJ, 618,155

Vaughan, S., \& Fabian, A. C. 2004, MNRAS, 348, 1415

Yaqoob, T., \& Padmanabhan, U. 2004, ApJ, 604, 63

Yaqoob, T., \& Serlemitsos, P. 2005, ApJ, 623, 112

Young, A. J., Lee, J. C., Fabian, A. C., Reynolds, C. S., Gibson, R. R., \& Canizares, C. R. 2005, ApJ, 631, 733

Weaver, K. A., Krolik, J. H., \& Pier, E. A. 1998, ApJ, 498, 213

Weaver, K. A., Yaqoob, T., Mushotzky, R. F., Nousek, J., Hayashi, I., \& Koyama, K. 1997, ApJ, 474, 675

Weaver, K. A. 2001, in ASP Conf. Ser. 249, The Central Kiloparsec of Starbursts and AGN: The La Palma Connection, ed. J. H. Knapen, J. E. Beckman, I. Shlosman, \& T. J. Mahoney (San Francisco: ASP), 389

Wilms, J., Allen, A., \& McCray, R. 2000, ApJ, 542, 914

Wandel, A., \& Mushotzky, R. F. 1986, ApJ, 306, L61 


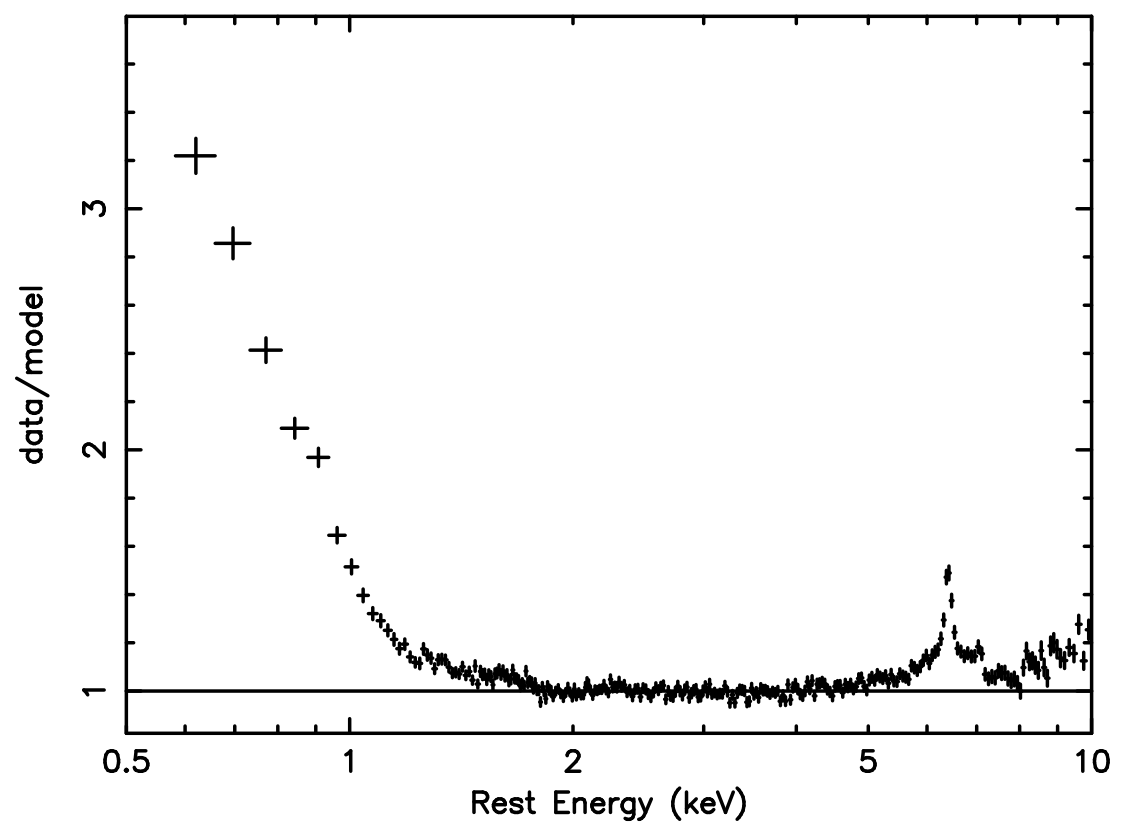

Fig. 1. - Ratio of the XMM-Newton pn data and the model when fitting an absorbed and redshifted power law over the $2-10 \mathrm{keV}$ band ignoring the iron emission line energy range $(5.5-7.5 \mathrm{keV})$. The photon index of the power law has been fixed to 1.8. Two different residuals are clearly present: a soft X-ray excess below $\sim 2 \mathrm{keV}$; and the Fe complex at $\sim 6.4$ $\mathrm{keV}$. 


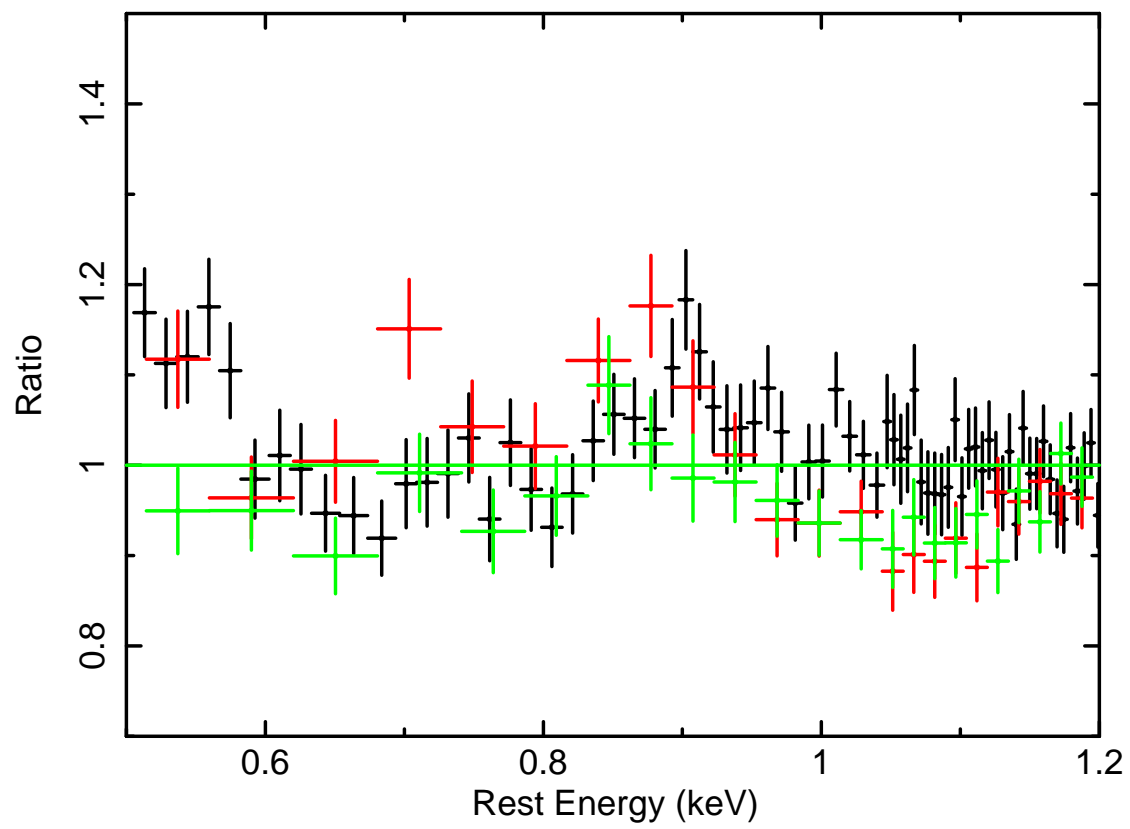

Fig. 2.- The ratio between the XMM-Newton pn (black points), MOS1 (red), and MOS2 (green) data and the the model, plotted between $0.5-1.5 \mathrm{keV}$. The model consists of the baseline absorbed power-law described in the text and an additional soft $(\Gamma=3)$ power law, absorbed only by a Galactic column. An emission line is clearly detected at $0.9 \mathrm{keV}$. 

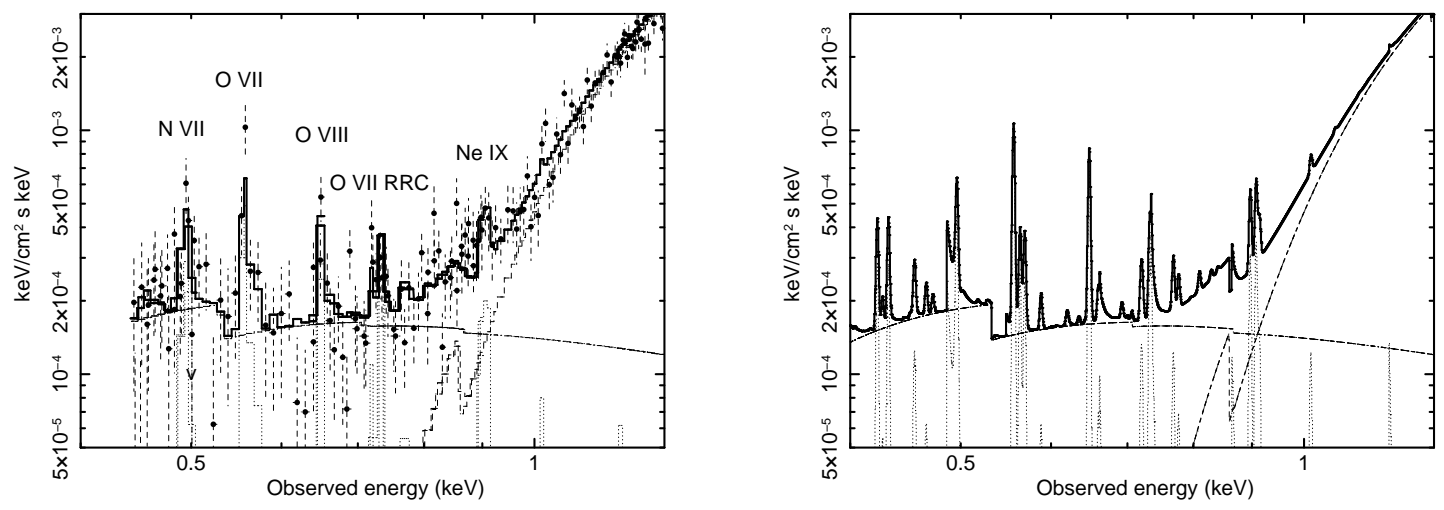

Fig. 3.- Left panel: XMM-Newton RGS1 and RGS2 spectra in the $0.4-1.3 \mathrm{keV}$ energy range. The underlying AGN continuum model is composed of an absorbed power law plus a scattered soft power-law component $(\Gamma=1.82)$. Several emission lines are clearly detected. The possible identifications of the 5 brightest lines are also shown. Above $\sim 0.9 \mathrm{keV}$ a steep rise of the continuum is evident, due to the emergence of the absorbed power-law component. Right panel: best fit photoionized plasma model (in the $0.4-1.3 \mathrm{keV}$ ) which includes a XSTAR component with an ionization parameter $\log \xi=1.29_{-0.13}^{+0.17}$ (see Section 3.2). 


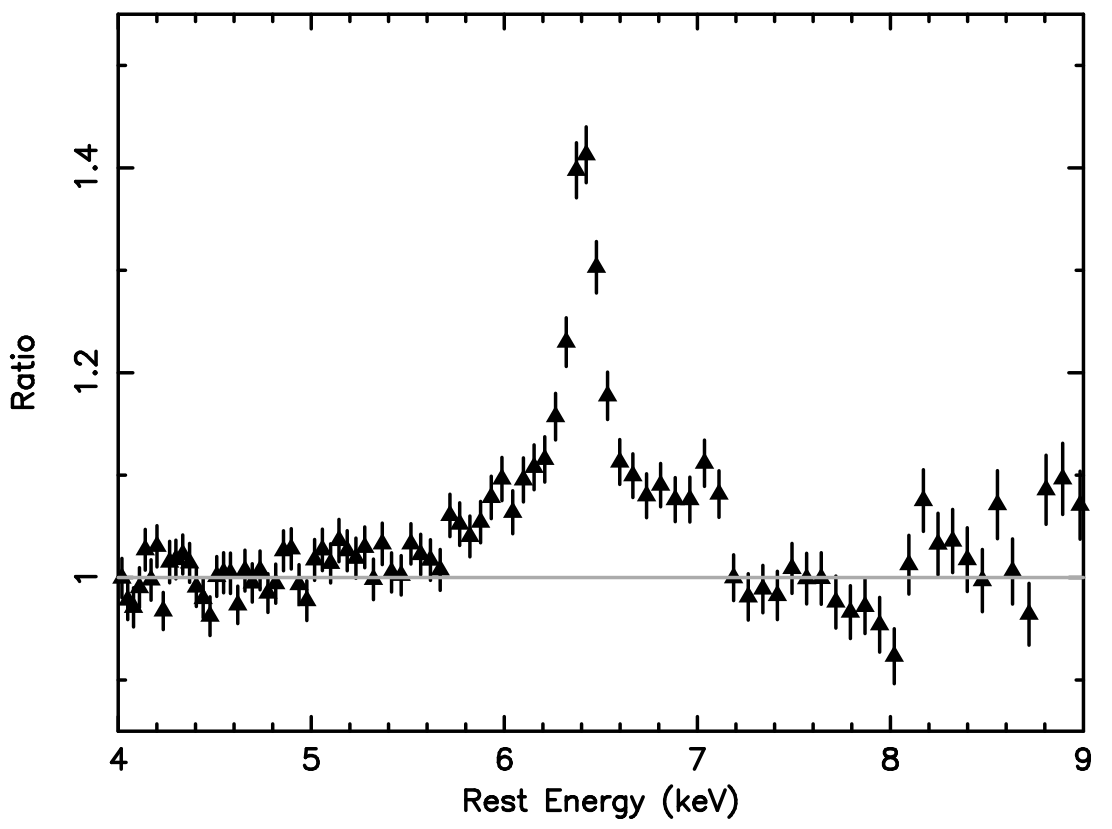

Fig. 4.- Ratio between the pn data and the absorbed power-law model $(\Gamma=1.65)$ showing the iron line profile. The data clearly show a narrow core at $6.4 \mathrm{keV}$, red and blue wings extending from $5.7 \mathrm{keV}$ to $7 \mathrm{keV}$ and a narrow emission feature at $\sim 7.05 \mathrm{keV}$, which is due to $\mathrm{Fe} \mathrm{K} \beta$. A sharp drop is also present at $\sim 7.1 \mathrm{keV}$ due to presence of reflection. 


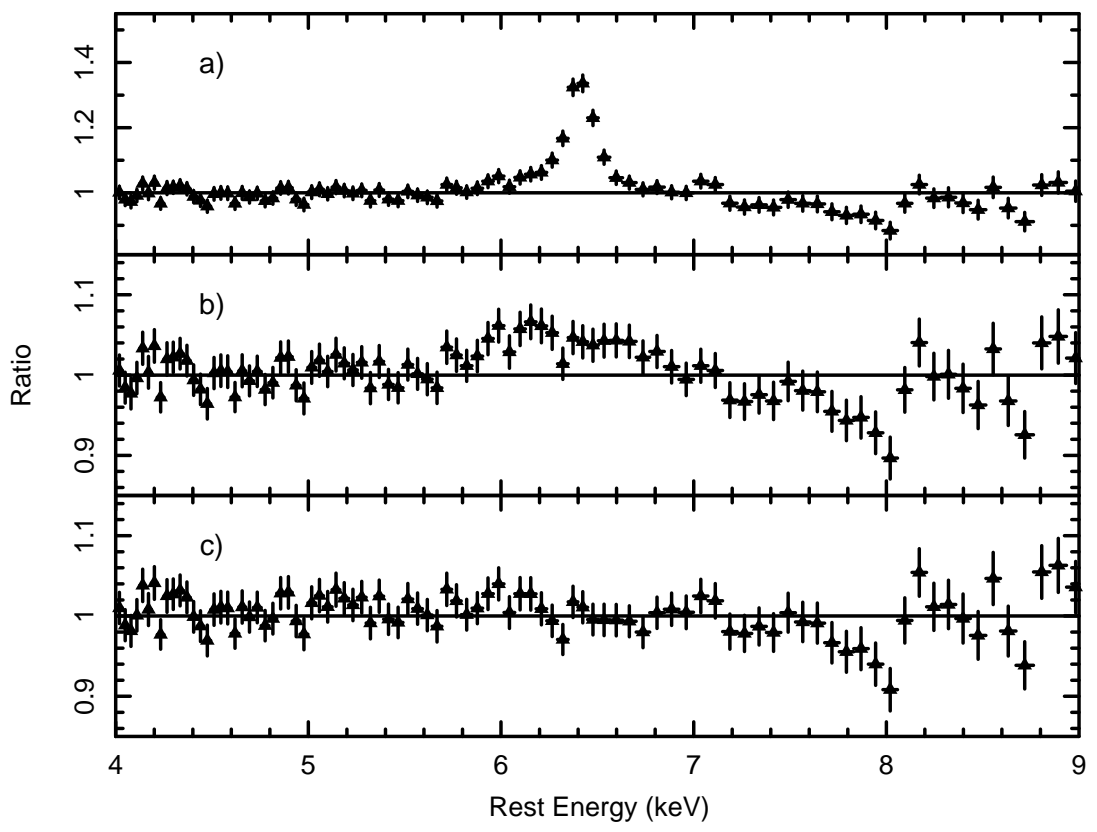

Fig. 5. - Data/model residuals for the pn data at the Fe K band. Panel (a) shows the data/model ratio when the underlying continuum is composed of an absorbed power law plus a reflection component $(R=1.1)$. Panel $(\mathrm{b})$ shows the residuals left when the narrow $\mathrm{Fe} \mathrm{K} \alpha$ and $\mathrm{Fe} \mathrm{K} \beta$ lines are added to the model. An excess between $5.8 \mathrm{keV}$ and $7 \mathrm{keV}$ is still present. Panel (c) shows the residuals when the broad component of the Fe K $\alpha$ line is fitted with a diskline model. The overall fit is now good and only a weak absorption feature is left at $8 \mathrm{keV}$. 


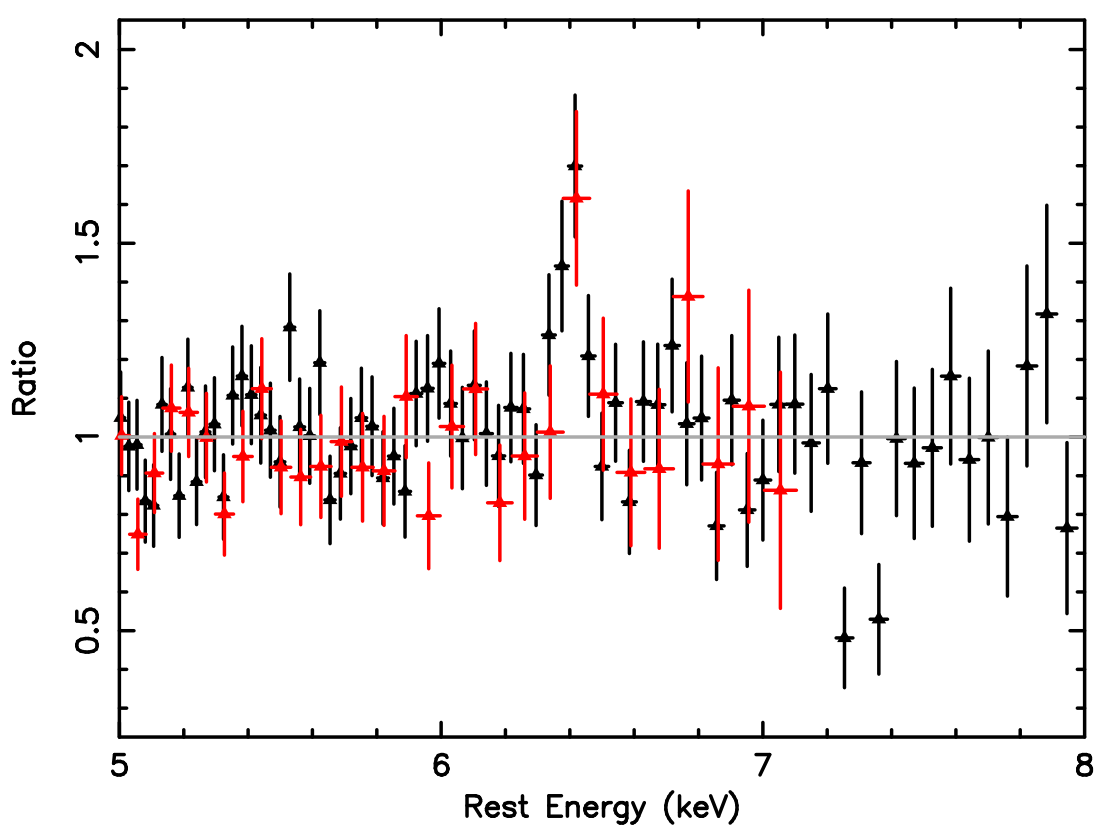

Fig. 6. - Data/model ratio for the Chandra HETG. The AGN continuum is composed of an absorbed power law plus a reflection component $(\Gamma=1.82 ; R=1.1)$ A strong narrow core at $6.4 \mathrm{keV}$ is observed. When modeled with a single Gaussian, the line is found to have $E W \sim 80 \mathrm{eV}$ and a width $\sigma=32_{-16}^{+19} \mathrm{eV}$. 


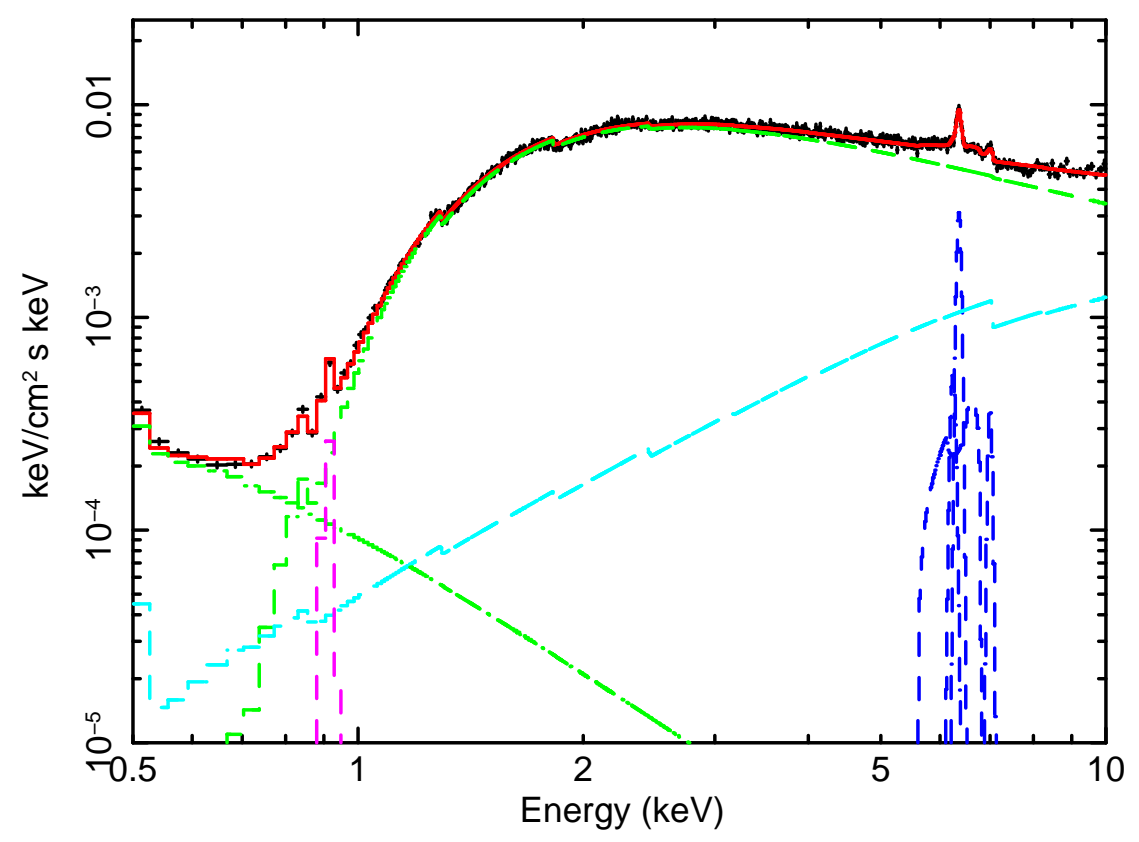

Fig. 7.-XMM-Newton spectrum of MCG-5-23-16. The black points denote the EPIC-pn data. The red line is the total best fit model. The AGN continuum model is composed of: an absorbed and a scattered power-law components (green lines online version) plus a reflection component (light blue line online version). The Fe K line complex (blue line online version) is composed of narrow and broad Fe $\mathrm{K} \alpha$ lines and a narrow $\mathrm{K} \beta$ line. A soft X-ray emission line at $\sim 0.9 \mathrm{keV}$ is also shown (magenta line online version). 


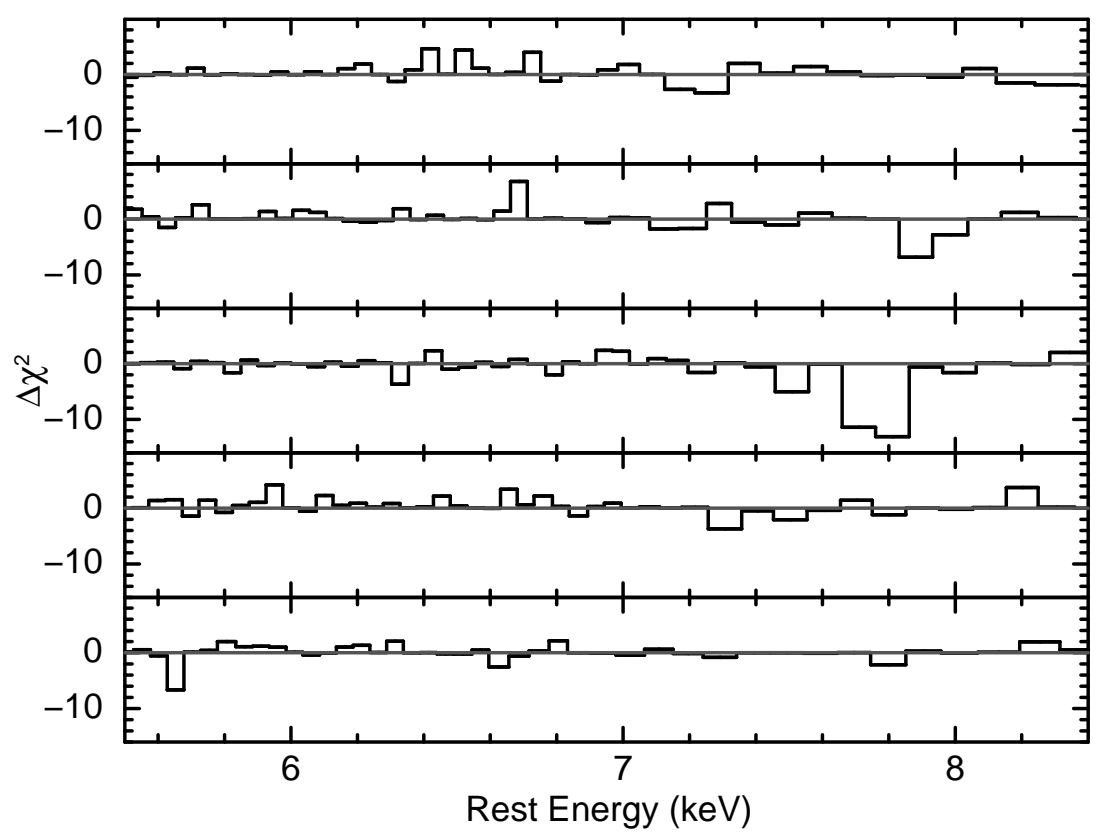

Fig. 8. - Contribution to the $\chi^{2}$ in the $5.5-8.4 \mathrm{keV}$ for the five pn spectra extracted with a time bin of $20 \mathrm{ksec}$. The Fe K $\alpha$ line has been parameterized with two Gaussian lines; all the parameters of the model except the primary power-law normalization have been kept tied together. The only strong deviation in $\Delta \chi^{2}$ is present in the third spectrum at $7.7 \mathrm{keV}$. 


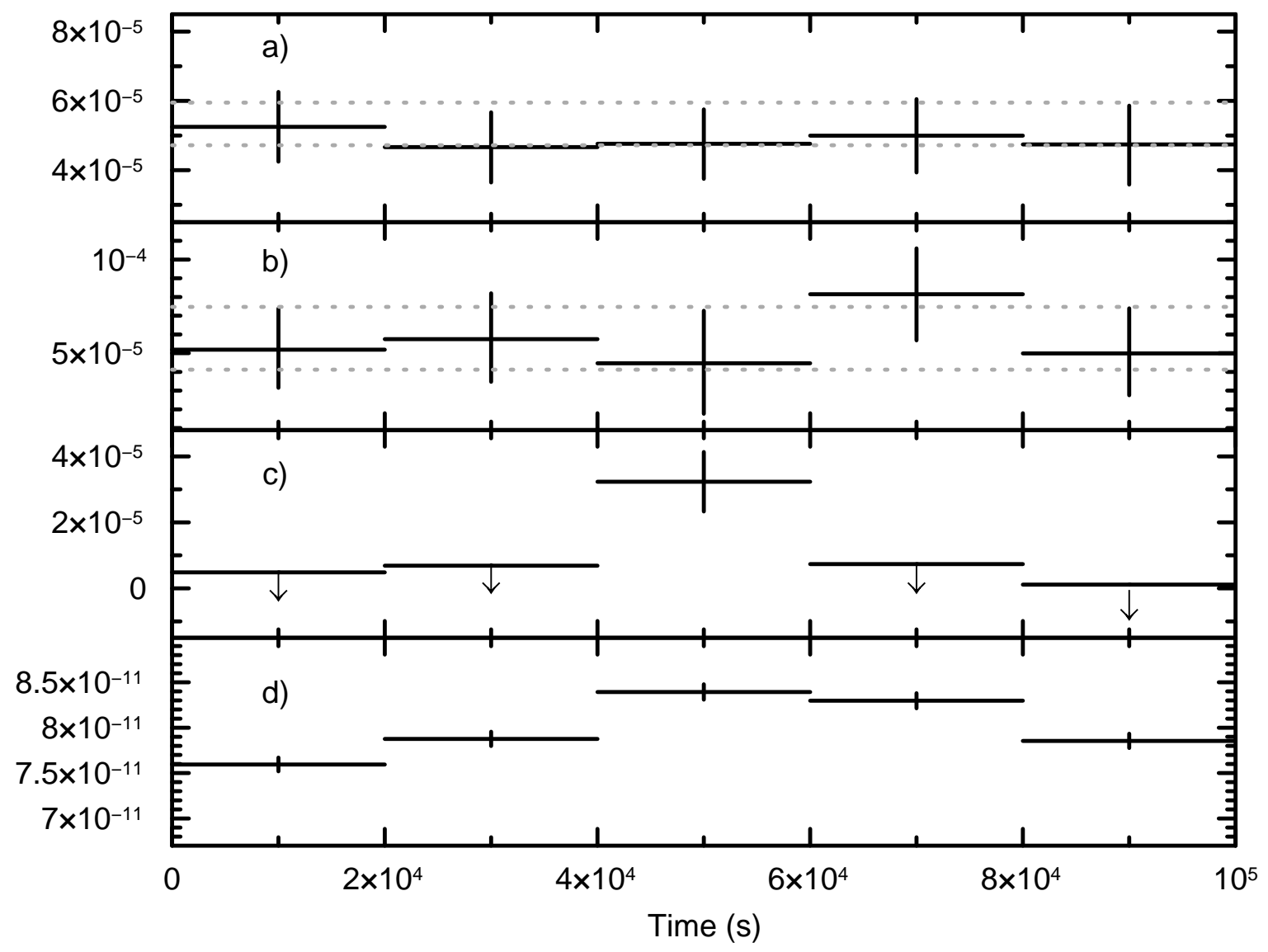

Fig. 9.- Time resolved spectral analysis. Panel (a): Fe $6.4 \mathrm{keV}$ narrow core intensity (in unit of photons $\mathrm{cm}^{-2} \mathrm{~s}^{-1}$ ) versus the time intervals. The dashed lines correspond to the $90 \%$ confidence level of the normalization of the narrow core measured in the average spectrum. Panel (b): same as panel a) for the broad component. Panel (c): Absolute intensity of the absorption feature (in unit of photons $\mathrm{cm}^{-2} \mathrm{~s}^{-1}$ ), the line energy has been fixed to the best fit value found for the third interval $(7.71 \mathrm{keV}$ ). Panel (d): MCG-5-23-16 2-10 keV flux (in erg $\left.\mathrm{cm}^{-2} \mathrm{~s}^{-1}\right)$. Error bars and upper limits are at the $90 \%$ confidence level. 


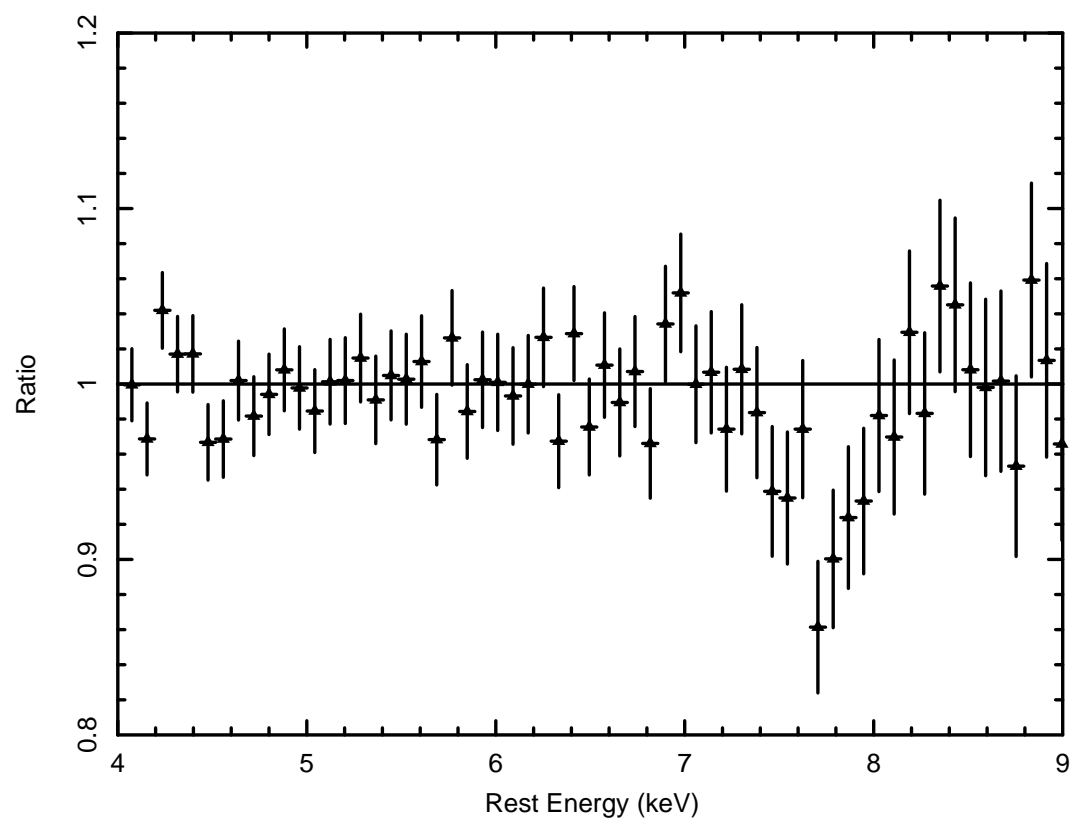

Fig. 10.- Data/model ratio of the pn spectrum of the third $20 \mathrm{ksec}$ interval, with the data binned with a constant energy bin of $80 \mathrm{eV}$. A deep absorption feature is visible at $7.7 \mathrm{keV}$ (see text for details). 


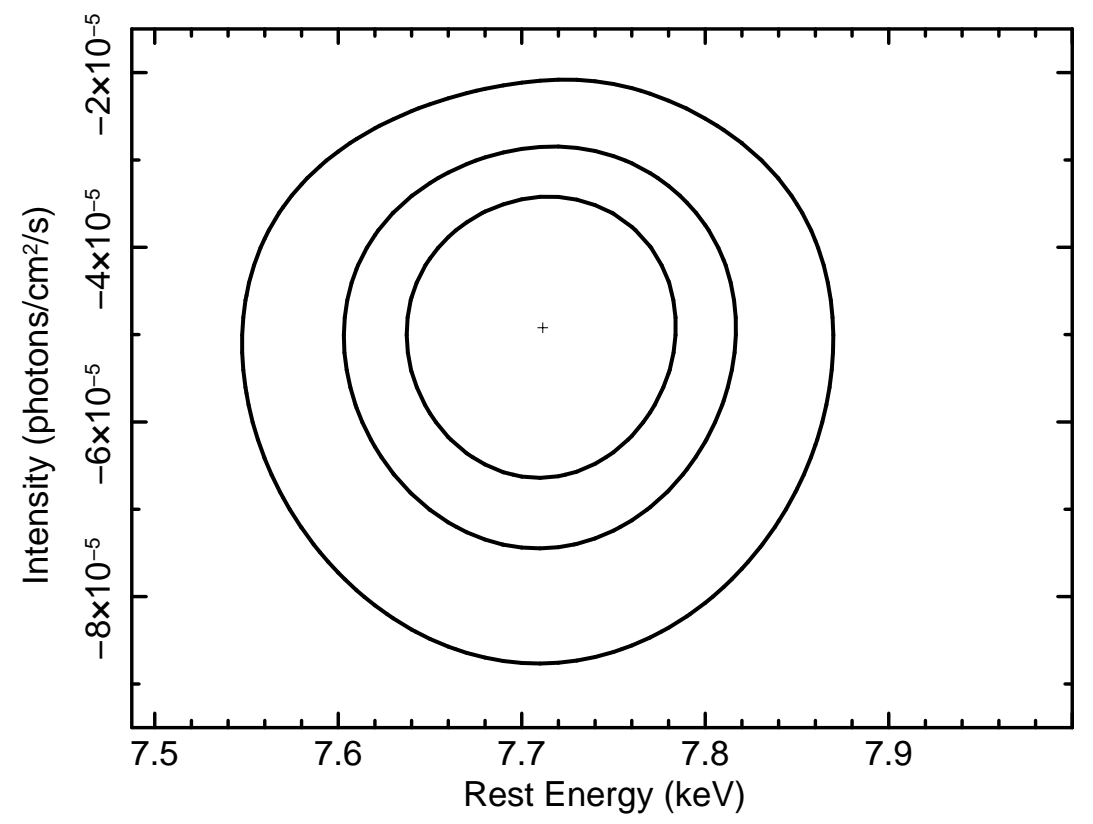

Fig. 11.- Derived 99\%, 90\% and 68\% confidence contours of the absorption line intensity vs. the observer-frame energy. 

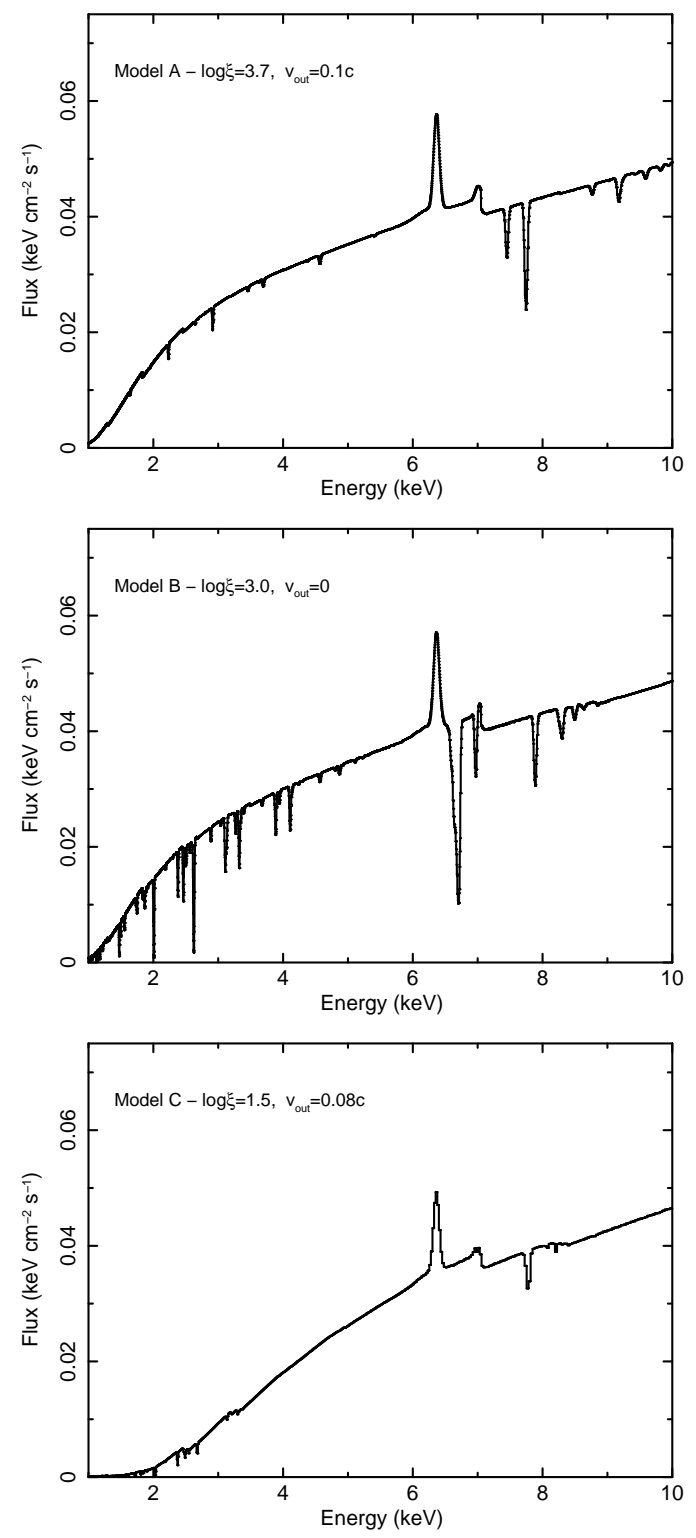

Fig. 12. - Example of high ionization warm absorber models. Panel(a) shows the best fit model: an ionization state characterized by $\log \xi=3.7_{-0.3}^{+0.2} \mathrm{erg}_{\mathrm{cm} \mathrm{s}}^{-1}$ and a column density of $\sim 8 \times 10^{22} \mathrm{~cm}^{-2}$ with an outflow velocity of $0.09 \pm 0.01 c$. The Fexxvi Ly $\alpha$ absorption feature shifted to $7.7 \mathrm{keV}$. fits well the observed dip at this energy. Panel (b): the absorption feature at $7.7 \mathrm{keV}$ can be accounted for by assuming a column density of $N_{\mathrm{H}}=10^{23} \mathrm{~cm}^{-2}$ and an ionization parameter of $\log \xi=3.0 \mathrm{erg} \mathrm{cm} \mathrm{s}^{-1}$. However, the model predicts stronger absorption due to the Fe XxV 1s-2p absorption line at $6.7 \mathrm{keV}$, which is not detected. The model in panel (c) shows that lower ionization $\mathrm{K} \beta$ absorption line without strong $\mathrm{K} \alpha$ from the same species, can be obtained assuming a column density of $N_{\mathrm{H}}=10^{23} \mathrm{~cm}^{-2}$, an ionization parameter of $\log \xi=1.5 \mathrm{erg} \mathrm{cm} \mathrm{s}^{-1}$. In this scenario a blueshift of $\sim 0.08 c$ would still be required to model the absorption line at $7.7 \mathrm{keV}$ in the spectrum and the column density required to model the $\mathrm{EW}$ of the $\mathrm{K} \beta$ absorption feature is $N_{\mathrm{H}}>>10^{23} \mathrm{~cm}^{-2}$; which would introduce too much continuum absorption below $6 \mathrm{keV}$, inconsistent with the pn data. 
Table 1. Log of the observations and exposure times.

\begin{tabular}{|c|c|c|c|c|c|}
\hline Mission & Instrument & $\mathrm{T}_{(\text {total })}(\mathrm{ks})$ & $\mathrm{T}_{(\mathrm{net})}(\mathrm{ks})$ & $\mathrm{T}_{\text {START }}$ & $\mathrm{T}_{\mathrm{STOP}}$ \\
\hline$X M M-N e w t o n$ & $\mathrm{PN}$ & 131.5 & 96.2 & 08/12/2005 21:11:28 & 10/12/2005 09:17:08 \\
\hline XMM-Newton & MOS1 & 131.5 & 101.6 & 08/12/2005 20:42:51 & 10/12/2005 09:16:48 \\
\hline$X M M-N e w t o n$ & MOS2 & 131.5 & 102.8 & 08/12/2005 20:43:51 & 10/12/2005 09:16:53 \\
\hline$X M M-N e w t o n$ & RGS1 & 131.6 & 97.2 & 08/12/2005 20:41:37 & 10/12/2005 09:18:03 \\
\hline$X M M-N e w t o n$ & RGS2 & 131.6 & 97.2 & 08/12/2005 20:41:42 & 10/12/2005 09:18:03 \\
\hline Chandra & ACIS-S HETG & 30 & - & 08/12/2005 17:41:30 & 09/12/2005 02:33:59 \\
\hline Chandra & ACIS-S HETG & 20 & - & 09/12/2005 20:52:11 & 10/12/2005 03:00:10 \\
\hline
\end{tabular}

Note. - For XMM-Newton the exposure values reported are total and net exposure time after filtering for high-background time intervals. 
Table 2. XMM-Newton RGS1 and RGS2: best fit emission lines required fitting with a photoionization model.

\begin{tabular}{|c|c|c|c|c|c|}
\hline $\begin{array}{c}\text { Energy } \\
(\mathrm{keV}) \\
(1)\end{array}$ & 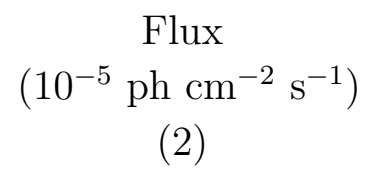 & $\begin{array}{l}\text { ID } \\
(3)\end{array}$ & $\begin{array}{l}\text { EW } \\
(\mathrm{eV}) \\
(4)\end{array}$ & $\begin{array}{l}\Delta C \\
(5)\end{array}$ & $\begin{array}{c}\mathrm{E}_{\mathrm{Lab}} \\
(\mathrm{keV}) \\
(6)\end{array}$ \\
\hline $0.499_{-0.001}^{+0.001}$ & $0.68_{-0.29}^{+0.39}$ & N VII Ly $\alpha$ & $10 \pm 5$ & 14.3 & 0.500 \\
\hline $0.564_{-0.002}^{+0.002}$ & $2.30_{-1.05}^{+1.42}$ & O vir He $\alpha$ & $46.5_{-20.7}^{+28.3}$ & 22.5 & $\begin{array}{l}0.561(\mathrm{f}) \\
0.569(\mathrm{i}) \\
0.574(\mathrm{r})\end{array}$ \\
\hline $0.653_{-0.001}^{+0.001}$ & $0.61_{-0.29}^{+0.37}$ & O viII Ly $\alpha$ & $14.7_{-7.0}^{+9.0}$ & 15.4 & 0.654 \\
\hline $0.732_{-0.007}^{+0.007}$ & $0.54_{-0.33}^{+0.40}$ & O vir RRC & $15.7_{-10.0}^{+11.0}$ & 7.0 & $>0.739$ \\
\hline $0.903_{-0.020}^{+0.020}$ & $0.42_{-0.28}^{+0.26}$ & Ne IX He $\alpha$ & $15.9 \pm 10.0$ & 10.4 & $\begin{array}{l}0.905(\mathrm{f}) \\
0.915(\mathrm{i}) \\
0.922(\mathrm{r})\end{array}$ \\
\hline
\end{tabular}

Note. - The model of underlying AGN continuum has been parametrized according to the best fit model obtained with the analysis of the pn spectrum (section 3). The $\Gamma$ of the soft power law is tied to the hard power-law component and fixed to the best fit value $(\Gamma=1.82)$. The energy of the lines are quoted in the rest frame. Fluxes and possible identifications are reported in col. (2) and (3) respectively. The EWs are reported in col. (4) and they are calculated against the soft power law component. In col. (5) the improvement of fit is shown using the $C$-statistic; the value for the model with no lines is $C=455.7$ for 358 PHA bins. In col. (6) we report the theoretical value for the transition energies. 
Table 3. Results of the fit for the mean spectrum and the low and high flux states.

\begin{tabular}{|c|c|c|c|c|}
\hline & Parameter & Mean & High & Low \\
\hline \multirow[t]{3}{*}{ Continuum } & $\Gamma$ & $1.82 \pm 0.01$ & $1.84 \pm 0.01$ & $1.81 \pm 0.01$ \\
\hline & $\mathrm{N}_{\mathrm{H}}^{a}$ & $1.49 \pm 0.01$ & $1.50 \pm 0.02$ & $1.49 \pm 0.02$ \\
\hline & Flux $^{b}$ & 8.16 & 8.84 & 7.39 \\
\hline \multirow[t]{3}{*}{ Narrow Gaussian } & $\mathrm{E}$ & $6.42 \pm 0.01$ & $6.41 \pm 0.02$ & $6.40 \pm 0.02$ \\
\hline & EW & $61_{-7}^{+7}$ & $48_{-13}^{+12}$ & $81_{-15}^{+15}$ \\
\hline & $\mathrm{N}^{c}$ & $5.4_{-0.6}^{+0.6}$ & $4.6_{-1.2}^{+1.3}$ & $6.5_{-1.2}^{+1.2}$ \\
\hline \multirow[t]{4}{*}{ Broad Gaussian } & $\mathrm{E}$ & $6.4^{f}$ & $6.4^{f}$ & $6.4^{f}$ \\
\hline & $\sigma$ & $0.35 \pm 0.1$ & $0.35_{-0.13}^{+0.15}$ & $0.37_{-0.13}^{+0.22}$ \\
\hline & EW & $64_{-16}^{+18}$ & $61_{-23}^{+17}$ & $75_{-32}^{+32}$ \\
\hline & $\mathrm{N}^{c}$ & $5.9 \pm 1.5$ & $5.9_{-2.6}^{+2.7}$ & $6.3_{-2.6}^{+2.9}$ \\
\hline \multirow[t]{4}{*}{ Diskline } & $\mathrm{R}_{\text {in }}$ & $48_{-20}^{+62}$ & $\ldots$ & $\ldots$ \\
\hline & $i$ & $41_{-12}^{+29}$ & $\ldots$ & $\ldots$ \\
\hline & EW & $53_{-13}^{+14}$ & $\ldots$ & $\ldots$ \\
\hline & $\mathrm{N}^{c}$ & $4.6 \pm 1.2$ & $\ldots$ & $\ldots$ \\
\hline
\end{tabular}

Note. - The line energies are expressed in units of keV, while theirwidths $\sigma$ and EWs are in eV. The disk radial emissivity has been fixed to $q=3$.

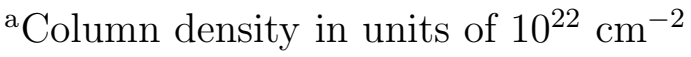

${ }^{\mathrm{b}} 2-10 \mathrm{keV}$ flux in units of $10^{-11} \mathrm{erg} \mathrm{cm}^{-2} \mathrm{~s}^{-1}$

${ }^{\mathrm{c}}$ Normalization of the Fe line in unit of $10^{-5}$ photons $\mathrm{cm}^{-2} \mathrm{~s}^{-1}$

${ }^{\mathrm{f}}$ Indicates that the parameter has been kept fixed. 
Table 4. Best fit parameters for the absorpion feauture detected at $7.7 \mathrm{keV}$ for the EPIC pn and MOS cameras and SUZAKU.

\begin{tabular}{lccc}
\hline \hline Mission-Instrument & $\begin{array}{c}\text { Energy } \\
(\mathrm{keV})\end{array}$ & $\begin{array}{c}\text { Flux } \\
\left(10^{-5} \mathrm{ph} \mathrm{cm}^{-2} \mathrm{~s}^{-1}\right)\end{array}$ & $\begin{array}{c}|E W| \\
(\mathrm{eV})\end{array}$ \\
\hline EPIC-pn $^{a}$ & $7.9 \pm 0.1$ & $-1.9 \pm 0.6$ & $33_{-11}^{+9}$ \\
EPIC-pn $^{b}$ & $7.7 \pm 0.1$ & $-3.2 \pm 0.9$ & $52 \pm 15$ \\
EPIC-MOS $^{a}$ & $7.4 \pm 0.2$ & $-1.1 \pm 1.0$ & $16 \pm 14$ \\
EPIC-MOS $^{b}$ & $7.4 \pm 0.2$ & $-2.2 \pm 1.8$ & $27 \pm 23$ \\
Suzaku-XIS0-1-2-3 $^{a}$ & $7.8 \pm 0.1$ & $-1.8 \pm 0.9$ & $30 \pm 15$ \\
Suzaku-XIS0-1-2-3 $^{b, c}$ & $7.65 \pm 0.4$ & $-3.5 \pm 3.0$ & $52 \pm 44$ \\
\hline
\end{tabular}

Note. - The parameters of the absorption line are derived adding an inverted gaussian component to the best fit model.

a Parameters refers to mean spectrum

${ }^{\mathrm{b}}$ Parameters are derived for the $20 \mathrm{ksec}$ time interval where the line is detected.

${ }^{\mathrm{c}}$ The net exposure of this Suzaku spectum is only $\sim 6 \mathrm{ksec}$ 